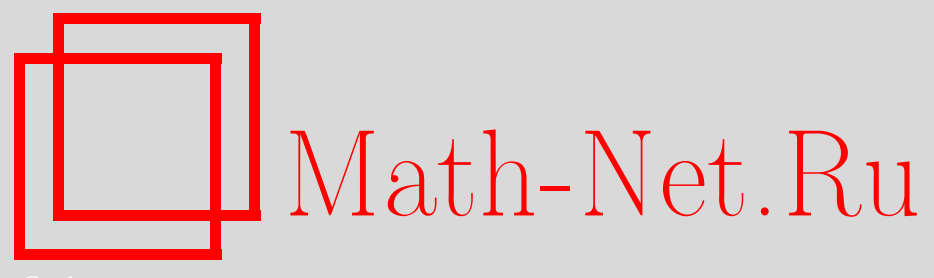

С. Б. Гашков, И. С. Сергеев, Об одном методе получения нижних оценок сложности монотонных арифметических схем, вычисляющих действительные многочлены, Матем. сб., 2012, том 203, номер 10, 33-70

DOI: https://doi.org/10.4213/sm7904

Использование Общероссийского математического портала Math-Net.Ru подразумевает, что вы прочитали и согласны с пользовательским соглашением http://www . mathnet.ru/rus/agreement

Параметры загрузки:

IP : 52.205 .19 .152

26 апреля 2023 г., 10:54:50

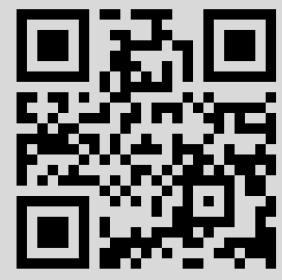




\author{
С. Б. Гашков, И. С. Сергеев
}

\title{
Об одном методе получения нижних оценок сложности монотонных арифметических схем, вычисляющих действительные многочлены
}

В работе предлагается метод получения нижних оценок сложности многочленов с положительными действительными коэффициентами при реализации схемами из функциональных элементов над монотонным арифметическим базисом $\{x+y, x \cdot y\} \cup\left\{a \cdot x \mid a \in \mathbb{R}_{+}\right\}$. Этот метод применяется к получению нескольких новых результатов. В частности, построены примеры многочленов степени $m-1$ по каждой из $n$ переменных с коэффициентами 0 и 1 , имеющих при $m^{n} \rightarrow \infty$ аддитивную монотонную сложность $m^{(1-o(1)) n}$ и мультипликативную монотонную сложность $m^{(1 / 2-o(1)) n}$. В таком виде эти нижние оценки неулучшаемы.

Библиография: 72 названия.

Ключевые слова: нижние оценки сложности, арифметические схемы, редкие множества, монотонная сложность, перманент.

\section{§ 1. Введение}

Методы получения эффективных нижних оценок вычислительной сложности функций вызывают интерес в связи с известной проблемой $P \stackrel{?}{=} N P$, возникшей в результате работ С. Кука, Р. Карпа и Л. Левина в 1970-е гг. Для отрицательного решения этой проблемы (а именно это представляется наиболее правдоподобным, хотя и более трудно осуществимым) необходимо для некоторых вычислительных проблем доказать отсутствие разрешающего их быстрого алгоритма. Под быстрым алгоритмом понимается алгоритм, число операций в котором (т.е. его сложность) по порядку не превосходит $n^{c}$, где $n$ - размер входных данных в двоичной кодировке, а $c$ - некоторая константа, не зависящая от $n$. Обычно алгоритмы с такой оценкой сложности называют полиномиальными (а класс $P$ задач, разрешимых такими алгоритмами, называют "полиномиальным"). Формализация понятия алгоритма и его сложности, а также классов $P$ и $N P$ осуществляется на языке детерминированных и недетерминированных машин Тьюринга (см., например, [1]). Но ввиду известной связи между временной сложностью вычислений на машинах Тьюринга и сложностью вычисления булевых функций схемами в стандартном булевом

Работа выполнена при финансовой поддержке Российского фонда фундаментальных исследований (гранты № 11-01-00508 и № 11-01-00792-а) и программы фундаментальных исследований Отделения математических наук РАН "Алгебраические и комбинаторные методы математической кибернетики и информационные системы нового поколения" (проект "Задачи оптимального синтеза управляющих систем").

(C) С. Б. ГАшков, И. С. Сергеев, 2012 
базисе $\{x \& y, x \vee y, \neg x\}$ (см., например, [2]) для отрицательного решения проблемы $P \stackrel{?}{=} N P$ достаточно для некоторых булевых функций $n$ переменных (связанных с известными $N P$-полными проблемами) доказать отсутствие реализующих (вычисляющих) эти функции булевых схем, состоящих из $n^{O(1)}$ элементов, т.е. получить для сложности этих функций сверхполиномиальную нижнюю оценку.

Сейчас ситуация такова, что не известно даже ни одной нелинейной нижней оценки булевой сложности, т.е. оценки вида $n f(n)$, где $f(n) \rightarrow \infty$ для $n$-местной булевой функции (в связи с этим довольно активно развивается в рассматриваемой области направление, в котором занимаются поиском нетривиальных линейных нижних оценок). В то же время О.Б. Лупанов в своих известных работах (см., например, его учебник [3], или [2]) показал, что почти все $n$-местные булевы функции имеют сложность $(1+o(1)) 2^{n} / n$, асимптотически равную сложности са́мой сложной такой функции, сложность которой он называл функцией Шеннона. Значит, доля функций, имеющих, например, сложность $2^{n(1-\varepsilon)}$, стремится к нулю с ростом $n$. Поэтому существование сложных функций несомненно. Проблема в том, как явно (т.е. эффективно в разумно понимаемом смысле) указать такую функцию и доказать, что она имеет высокую сложность. Известны не вполне эффективные результаты о высоких нижних оценках сложности булевых функций (см., например, [2]), которые представляют интерес, но не дают решения проблемы эффективных нижних оценок.

О. Б. Лупанов и С. В. Яблонский еще в 1950-е гг. (задолго до появления “теории NP-полноты") осознавали важность проблемы нижних оценок. Так как для случая реализации булевых функций схемами общего вида она представлялась (и сейчас представляется) слишком трудной, то начались поиски методов получения нижних оценок сложности реализации булевых функций схемами с ограничениями. Ограничения накладывались как на структуру схем ${ }^{1}$, так и на базис, из элементов которого строились схемы ${ }^{2}$. В этом направлении еще в 1960-хгг. работали сам О.Б. Лупанов и его ученики и последователи (см., например, [2], [4]-[6]). За редкими исключениями все доказанные оценки были полиномиальными.

В 1984 г. А.А. Разборов и А.Е. Андреев независимо получили сверхполиномиальные нижние оценки сложности реализации булевых функций в монотонном базисе $\{\&, \bigvee\}$. В работах Разборова [7], [8] были получены оценки вида $n^{\Omega(\log n)}$ для монотонной сложности логического аналога перманента и для функции, распознающей в графе наличие клики данного размера ${ }^{3}$, в работах Андреева [11], [12] для специально построенной функции была получена почти экспоненциальная нижняя оценка вида $2^{n^{1 / 8-o(1)}}$. В дальнейшем он уточнил свою оценку до $2^{n^{1 / 4-o(1)}}$ (такую же оценку получили и в [10]), а в [9] получил для другой функции оценку $2^{n^{1 / 3-o(1)}}$. В [13], вероятно не зная о [9], Р. Раз и Д. Харник получили подобную же оценку.

\footnotetext{
${ }^{1}$ Например, рассматривалась реализация частным видом булевых схем - схемами без ветвления выходов элементов, т.е. формулами, рассматривались совсем другие виды схем - контактные, вентильные, контактно-вентильные схемы.

${ }^{2} \mathrm{~B}$ частности, рассматривались схемы над неполными базисами.

${ }^{3}$ Андреев [9] и независимо Н. Алон и Р. Боппана [10] усилили оценку для функции распознавания клики до $2^{n^{1 / 6-o(1)}}$.
} 
В некоторой степени аналогичными вопросам булевой сложности являются вопросы о сложности вычисления действительных (или комплексных) многочленов схемами (неветвящимися программами), элементы которых реализуют арифметические операции и константы. Сейчас такие схемы часто называют арифметическими схемами.

Для реализации действительных многочленов одной переменной степени $n$ можно применить схему Горнера. В. Я. Пан показал в начале 1960-х гг., что эта схема оптимальна, если в ней используются в качестве констант только коэффициенты реализуемого многочлена, но число умножений в нем можно уменьшить почти в два раза, если использовать в схеме любые константы. Тогда же было показано, что почти все многочлены нельзя вычислить существенно проще, чем методом Пана (см., например, [14]). Однако предъявить конкретный “трудновычислимый” многочлен оказалось непросто. Ф. Штрассен, его ученики и последователи, выполнили несколько работ на эту тему и построили в [15]-[18] примеры многочленов сложности $\Omega(n)$, аддитивной сложности $n$, мультипликативной сложности $n / 2+O(1)$, нескалярной сложности ${ }^{4}(\sqrt{n})$. В силу результатов Пана и Г. Г. Белаги (см. [14]) и М. Петерсона-Л. Стокмейера [19] все эти оценки неулучшаемы по порядку (а некоторые - с точностью до аддитивной константы). Обзор работ того времени по нижним оценкам в алгебраической теории сложности можно найти в [20], более современное состояние вопроса изложено в [21]. Но у указанных многочленов используются коэффициенты довольно сложной природы (либо быстро растущие с ростом номера коэффициента рациональные числа, либо комплексные корни из единицы быстро растущих степеней).

Существование многочленов сложности $\Omega(n / \log n)$ с коэффициентами 0 и 1 доказано пока неэффективно (К. Шнорром и Ш. ван де Вилем в [22] доказано, что почти все многочлены таковы). Для мультипликативной (и для нескалярной) сложности там же получена оценка $\Omega(\sqrt{n / \log n})$, а для аддитивной сложности - оценка $\Omega(\sqrt{n} / \log n)$. Известно [23], что сложность многочленов с коэффициентами 0, 1 не может превосходить по порядку $n / \log n$, даже если в схеме используется из всех возможных констант только константа 1 (и поэтому схема является монотонной), при этом мультипликативная сложность не превосходит $O(\sqrt{n})$. Многочлен с коэффициентами 0 и 1 , имеющий аддитивную монотонную сложность $n^{1-o(1)}$ и мультипликативную монотонную сложность $n^{1 / 2-o(1)}$, будет указан в настоящей работе (тем самым решается открытая проблема 9.3 из [21]).

Вопрос о сложности вычислений различных классов многочленов, коэффициенты которых принимают значения из данного конечного множества, рассматривался также в [24]-[26]. Для этого в базисе, над которым строятся схемы, достаточно иметь только конечное множество констант. В [25], [26] отмечалось, что для получения асимптотически оптимальных верхних оценок можно использовать модификацию упоминавшегося выше метода Лупанова. В частности, для многочленов от $n$ переменных, у которых степень по каждой переменной не больше 1 (такие многочлены в [27] назывались многочленами

\footnotetext{
${ }^{4}$ Так для краткости здесь и далее называем минимальное число умножений общего вида, без учета умножений на константы.
} 
Жегалкина ${ }^{5}$, а в современных зарубежных работах они называются мультилинейными) и имеющих только коэффициенты 0,1 , верхняя оценка монотонной сложности асимптотически равна $2^{n} / n$, а верхняя оценка мультипликативной сложности равна $O\left(2^{n / 2}\right)$, причем первая оценка асимптотически неулучшаема.

Мультилинейные многочлены от $n$ переменных можно путем замены переменных $x_{i}=x^{2^{i-1}}$ преобразовать без изменения множества коэффициентов в многочлен степени не больше $2^{n}-1$ от одной переменной, и наоборот: многочлен одной переменной преобразовать в мультилинейный. Отсюда видно, что из верхних оценок сложности мультилинейных многочленов следуют подобные же оценки для многочленов одной переменной (поэтому результаты [23] следуют из [25]), а из нижних оценок сложности многочленов одной переменной можно вывести подобные же оценки для мультилинейных многочленов. ${ }^{6}$ Поэтому из результатов И. фон цур Гатена-Штрассена, И. Хайнца-М. Зивекинга и Х. Штосса [15]-[17] можно вывести, что указанные абзацем выше оценки для мультилинейных многочленов по порядку нельзя существенно улучшить, даже используя схемы в полном базисе ${ }^{7}$. Для мультипликативной сложности класса всех мультилинейных многочленов с коэффициентами 0, 1 от $n$ переменных получается, например, нижняя оценка $\Omega\left(2^{n / 2} / \sqrt{n}\right)$. Можно также явно указать мультилинейные многочлены аддитивной сложности $2^{n}-1$, мультипликативной сложности $2^{n-1}+O(n)$, но их коэффициенты будут весьма сложной природы.

Для монотонных арифметических схем первая почти экспоненциальная эффективная нижняя оценка была получена Шнорром в [29]. Она имела вид по порядку $2^{\sqrt{n}} / \sqrt[4]{n}$, а точнее, для некоторого многочлена степени $n^{2}$ от $4 n^{2}$ переменных (соответствующего задаче о клике [1]) аддитивная монотонная сложность фактически равна биномиальному коэффициенту $C_{2 n}^{n}-1$.

Почти экспоненциальные нижние оценки вида $2^{\Omega(\sqrt{n})}$ монотонной сложности многочленов получены также Р. Джеррумом, М. Сниром, Л. Дж. Вэлиантом в [30], [31]. В [30] для монотонной мультипликативной сложности вычисления перманента ${ }^{8}$ действительной $(n \times n)$-матрицы получена асимптотически точная оценка $(1-o(1)) 2^{n-1} n$, соответствующая верхняя оценка была доказана Г. Райзером и В. Юркатом в 1967 г. (см., например, [14]). Как известно, определитель ${ }^{9}$ в полном базисе имеет сложность $O\left(n^{\omega+o(1)}\right)$, где $\omega-$ экспонента матричного умножения, про которую сейчас известно, что $2 \leqslant \omega<2,4$.

В [32], [33] Вэлиант указал на трудность получения почти экспоненциальных нижних оценок сложности вычисления некоторых монотонных многочленов в полном базисе. А именно он показал, что наличие такой оценки для

\footnotetext{
${ }^{5}$ В честь профессора МГУ И. И. Жегалкина, использовавшего подобные многочлены B [28].

6 Чтобы из схемы $S$, реализующей мультилинейный многочлен $n$ переменных, получить схему, реализующую соответствующий ему многочлен одной переменной, надо входы схемы $S$ присоединить к выходам схемы, вычисляющей набор степеней $x^{2^{i}}, i=0, \ldots, n-1,-$ такая схема строится из $n-1$ элементов умножения.

${ }^{7}$ То есть в базисе, содержащем вычитание или все возможные, в том числе и отрицательные, константы.

${ }^{8}$ Перманент - это мультилинейный монотонный многочлен степени $n$ от $n^{2}$ переменных с коэффициентами 0,1 .

${ }^{9}$ Определитель является мультилинейным немонотонным многочленом степени $n$ от $n^{2}$ переменных с коэффициентами $0, \pm 1$.
} 
перманента действительной матрицы означало бы, что $V N P \neq V P$, где проблема $V N P \stackrel{?}{=} V P$ - построенный Вэлиантом алгебраический аналог обычной (булевской) проблемы $P \stackrel{?}{=} N P$. Там же он показал, что вычисление перманента целочисленной матрицы по модулю $2^{k}$ можно выполнить булевыми схемами со сложностью $O\left(n^{4 k-3}\right)$.

Вместо перманента по причинам, которые объясняются, например, в [34], в развитой Вэлиантом теории алгебраических классов сложности удобнее рассматривать мультилинейный многочлен

$$
H C_{n}=\sum_{\sigma} \prod_{i=1}^{n} x_{i, \sigma(i)},
$$

распознающий гамильтоновость $n$-вершинного графа, где суммирование производится по всем циклическим перестановкам $\sigma \in S_{n}$. Подробное изложение теории Вэлианта имеется в [21]. Заметим еще, что аналог проблемы $P \stackrel{?}{=} N P$ для поля комплексных чисел изучался в [35].

К изучению сложности перманента и далее сохранялся интерес. В [36] методы [30] применены для получения таких же нижних оценок монотонной сложности $(0,1)$-перманента ${ }^{10}$. Подход, связанный с выражением перманента матрицы через детерминант матрицы большего размера, развивался в [37]. В [38] показано, что при реализации мультилинейными формулами ${ }^{11}$ в полном базисе и перманент, и детерминант имеют сверхполиномальную сложность.

С теорией классов сложности связаны некоторые способы введения понятия эффективности. Эффективность служит формализацией интуитивного представления о конкретности, явности (что значит, что функция задана явно?).

Например, эффективность (последовательностей) булевых функций часто отождествляют с принадлежностью их классу $N P$. Подобное определение, во всяком случае, до тех пор, пока не решена проблема $P \stackrel{?}{=} N P$, не кажется удачным. Ибо в случае $P=N P$ получение эффективных сверхполиномиальных нижних оценок сложности в полном базисе превращается в абсурд.

Явное задание многочленов может быть выполнено многими способами, например, конкретным указанием либо простым правилом вычисления коэффициентов при мономах или корней. Примеры сложных многочленов, которые строятся в настоящей статье, имеют коэффициенты 0 и 1. Они удовлетворяют, в частности, следующему определению эффективности. Многочлен является эффективным, если булева функция, вычисляющая коэффициент многочлена по степени монома (степень рассматривается в двоичном представлении), реализуется булевой схемой полиномиальной сложности над стандартным базисом. Из результата [33] следует, что многочлен, эффективный в этом смысле, принадлежит классу $V N P$ (алгебраический аналог класса $N P$ ).

Однако даже принадлежность классу $V N P$ нельзя признать вполне удовлетворительным критерием эффективности многочленов. Например, про такой конкретно заданный многочлен, как мультилинейный аналог многочлена

${ }^{10}$ То есть для вычисления перманента $(0,1)$-матрицы использовались монотонные многочлены, у которых значение при подстановке вместо переменных нулей или единиц всегда равно перманенту соответствующей $(0,1)$-матрицы.

${ }^{11} \mathrm{~B}$ мультилинейных формулах все подформулы реализуют мультилинейные многочлены. 
$\sum 2^{2^{k}} x^{k}$ (пример, предложенный Штрассеном) сложно сказать, принадлежит он $V N P$ или нет. Если все же принадлежит (это представляется менее вероятным), то $V P \neq V N P$, ввиду того, что сложность данного многочлена в полном арифметическом базисе сверхполиномиальна. Если не принадлежит, то, наверное, следует искать другое определение эффективности.

В 1983 г. О. М. Касим-Заде [39], [40] построил мультилинейный многочлен от $n$ переменных с коэффициентами 0,1 с аддитивной монотонной сложностью $2^{\lfloor n / 2\rfloor}-1$, впервые получив тем самым эффективную экспоненциальную нижнюю оценку для указанной меры сложности. Экспоненциальные нижние оценки сложности монотонных многочленов получил также в 1984 г. С. Е. Кузнецов [41] (как отмечено в [39]-[41], подобные оценки извлекаются уже из более ранней работы Кузнецова [42]). В 1987 г. [43] был предложен метод получения нижних оценок монотонной сложности, являющийся некоторым развитием идей [39], [40], [29], и с его помощью, в частности, эффективно указан мультилинейный многочлен от $n$ переменных с коэффициентами 0,1 с аддитивной монотонной сложностью, по порядку не меньшей $2^{2 n / 3}$, и с мультипликативной монотонной сложностью, по порядку не меньшей $2^{c n}$, где константа $c>1 / 3$.

В работе [40] Касим-Заде также построил последовательность мультилинейных многочленов $n$ переменных с рациональными коэффициентами, имеющих сложность $\Omega\left(2^{c_{1} n}\right)$ над монотонным арифметическим базисом и имеющих сложность $O\left(n^{c_{2}}\right)$ над базисом, дополненным вычитанием. ${ }^{12}$

Вероятно, не зная о работах [39]-[43], в недавней работе [44] Р. Раз и А. Иехудаев получили нижнюю оценку вида $2^{\Omega(n)}$ для монотонной сложности некоторого мультилинейного многочлена $n$ переменных. В [44] также получены некоторые результаты о сложности различного типа мультилинейных формул (т.е. формул в мультилинейных базисах). ${ }^{13}$

Некоторые методы получения нижних оценок для монотонной сложности булевых функций переносятся и на случай реализации булевых функций монотонными схемами над полем действительных чисел ${ }^{14}$ - этому посвящены, например, работы С. Кука-А. Хакена и П. Пудлака [48], [49]. В последней из них для монотонной булевой функции $n$ переменных, распознающей наличие клики данного размера в графе, получена нижняя оценка $2^{n^{1 / 6-o(1)}}$.

В работе [43] в силу ограниченности объема была приведена только схема доказательства основных теорем. Здесь мы приводим (в несколько обобщенном виде) метод получения нижних оценок из [43] вместе с полными доказательствами и несколько новых результатов.

\footnotetext{
${ }^{12}$ Результаты работ Шнорра и Касим-Заде [29], [39], [40] формулировались для вычислений над полем $\mathbb{Q}$, а не над $\mathbb{R}$, но это не принципиально.

${ }^{13}$ Согласно [44] модель мультилинейных схем и формул впервые появилась в [45] в 1995 г. Мы все же заметим, что в [27], [46] эти понятия появились еще в 1980 г. и там же получены некоторые результаты о сложности реализации булевых функций мультилинейными схемами и формулами. Работы [27], [46] получили продолжение в [47], где уже использовались термины мультилинейный многочлен и мультилинейная формула. Работы [47], [27] цитировались в [35].

14 Эти схемы состоят из элементов, реализующих монотонные функции действительных переменных. Схема реализует булеву функцию, если при значениях входов схемы, равных 0 или 1 , выход схемы тоже равен 0 или 1. Заметим, что такое определение реализации булевых функций схемами в непрерывных базисах было предложено Лупановым и исследовалось в [27], [46] в конце 70-х гг.
} 
В частности, путем приложения к упомянутому методу интересного комбинаторного результата Л. Роньяи, Я. Коллара и Т. Сабо [50], будет получена для конкретных многочленов $n$ переменных степени $m-1$ по каждой переменной с единичными коэффициентами нижняя оценка аддитивной монотонной сложности $m^{n(1-o(1))}$ и нижняя оценка мультипликативной монотонной сложности $m^{n(1 / 2-o(1))}$ при $m^{n} \rightarrow \infty$, которые в таком виде уже не поддаются улучшению. Этим примером охватываются важные частные случаи: мультилинейных многочленов $(m=2)$ и многочленов одной переменной $(n=1)$.

Также будет эффективно построен мультилинейный многочлен $n$ переменных степени $m-1$ по каждой переменной с единичными коэффициентами, для которого отношение сложности реализации в монотонном базисе к сложности реализации в полном базисе не меньше чем $m^{(0,5-o(1)) n}$ при $m^{n} \rightarrow \infty$. Ранее, рассматривая случай $m=2$ мультилинейных многочленов, в [31] Вэлиант получил для такого же отношения нижнюю оценку $2^{\Omega(\sqrt{n})}$, а Касим-Заде экспоненциальную оценку $2^{c n}$ (см. выше).

В качестве еще одного приложения упомянутого метода будет получена нижняя оценка $\Omega\left(2^{n} \sqrt{n}\right)$ аддитивной монотонной сложности перманента $n$-го порядка. Для упоминавшегося выше многочлена $H C_{n}$ аналогичным образом можно получить нижнюю оценку $\Omega\left(2^{n} / \sqrt{n}\right)$. (Авторам не известны какие-либо другие результаты об аддитивной монотонной сложности указанных многочленов.)

Будет также показано, что нижние оценки, полученные предложенным в этой статье методом, существенно улучшены быть не могут.

\section{§ 2. Основные определения}

Будем рассматривать многочлены, вычисляемые схемами, состоящими из элементов сложения, умножения и положительных действительных констант. Для любого такого многочлена $f$ обозначим через $L_{+}(f)$ наименьшее число сложений, а через $L_{\times}(f)$ - наименьшее число нескалярных умножений, необходимое для его вычисления. Как обычно, полукольцо монотонных многочленов с переменными $x_{1}, \ldots, x_{n}$ относительно операций сложения и умножения обозначается через $\mathbb{R}_{+}\left[x_{1}, \ldots, x_{n}\right]$. Обозначим через $P\left(N^{n}\right)$ полукольцо конечных подмножеств множества $N^{n}$ (где $N=\mathbb{N} \cup\{0\}$ ) относительно операции дизъюнкции $\vee$ и умножения $\times$ : если $A, B \in P\left(N^{n}\right)$, то $A, B \subset N^{n}$,

$$
A \vee B=A \cup B, \quad A \times B=\{a+b \mid a \in A, b \in B\} .15
$$

Через mon обозначим гомоморфизм полукольца $\mathbb{R}_{+}\left[x_{1}, \ldots, x_{n}\right]$ в полукольцо $P\left(N^{n}\right)$, определяемый условием: $a=\left(a_{1}, \ldots, a_{n}\right) \in \operatorname{mon} f$ тогда и только тогда, когда многочлен $f$ содержит моном $c_{a} x_{1}^{a_{1}} \cdots x_{n}^{a_{n}}$.

\footnotetext{
${ }^{15}$ Множество $A \times B$ является суммой Минковского множеств $A$ и $B$. В настоящей работе сумму Минковского вынужденно приходится обозначать через “х”, поскольку она играет роль умножения (так преобразуются показатели степеней мономов при перемножении монотонных многочленов).
} 
То, что отображение mon действительно является гомоморфизмом, следует из непосредственно проверяемых равенств

$$
\begin{aligned}
\operatorname{mon}\left(f_{1}+f_{2}\right)= & \operatorname{mon} f_{1} \vee \operatorname{mon} f_{2}, \quad \operatorname{mon}\left(f_{1} f_{2}\right)=\operatorname{mon} f_{1} \times \operatorname{mon} f_{2}, \\
& \operatorname{mon} 0=\varnothing, \quad \operatorname{mon} 1=\{(0, \ldots, 0)\} .
\end{aligned}
$$

Подмножество $H$ полугруппы $(G,+)$ назовем $(k, l)$-редкuм, если оно не содержит подмножеств вида $A \times B=\{a+b \mid a \in A, b \in B\}$, где $|A|=k$ и $|B|=l$ (здесь и далее мощность конечного множества $M$ обозначается через $|M|$ ).

В случае, когда $(G,+)$ - группа, это определение эквивалентно следующему: подмножество $H$ группы $(G,+)$ называется $(k, l)$-редким, если для любых различных элементов $g_{1}, \ldots, g_{k} \in G$ справедливо неравенство

$$
\left|\bigcap_{i=1}^{k} g_{i} H\right|<l, \quad g_{i} H=\left\{g_{i}\right\} \times H .
$$

Эквивалентность двух определений проверяется непосредственно. Действительно, пусть для некоторых $g_{1}, \ldots, g_{k} \in G$ выполняется $\left|\bigcap_{i=1}^{k} g_{i} H\right| \geqslant l$. Поскольку $\left\{-g_{1}, \ldots,-g_{k}\right\} \times \bigcap_{i=1}^{k} g_{i} H \subset H$, то $H$ не является $(k, l)$-редким в смысле первого определения.

Наоборот, пусть $A \times B \subset H, A=\left\{g_{1}, \ldots, g_{k}\right\},|B|=l$. Поскольку $B \subset$ $\bigcap_{i=1}^{k}\left(-g_{i}\right) H$, то $\left|\bigcap_{i=1}^{k}\left(-g_{i}\right) H\right| \geqslant l$. Значит, $H$ не является $(k, l)$-редким в смысле второго определения.

Далее, используя понятие $(k, l)$-редкого подмножества в коммутативных полугруппах, в частности, в $\left(N^{n},+\right)$, будем всегда полагать $k \leqslant l$. Кроме того, $(k, k)$-редкое подмножество будем называть сокращенно $k$-редким.

\section{§ 3. Основная теорема}

Обозначим через $\alpha(k)$ наибольшее количество различных булевых $(k-1)$ мерных векторов, ни один из которых не равен дизъюнкции двух других. Легко проверить, что $\alpha(2)=2, \alpha(3)=3, \alpha(4)=5, \alpha(5)=9$ и $\alpha(k)<2^{k-2}$ при $k>5$. В [51] Д. Клейтмен показал, что $\alpha(k) \sim C_{k-1}^{\lfloor(k-1) / 2\rfloor}$.

Следующая теорема была сформулирована и доказана для случая $k=l$ в [43].

Теорема 1. Пусть $k>1 u$ mon $f-(k, l)$-редкое подмножество множества $\left(N^{n},+\right)$. Положим $h=\max \left\{|A|\left|A=A_{1} \times \cdots \times A_{p} \subset \operatorname{mon} f,\right| A_{i} \mid<l\right\}$ u $H=h^{-1}|\operatorname{mon} f|$. Тогда справедливы неравенства:

(i) $L_{+}(f) \geqslant H-1$;

(ii) $L_{\times}(f) \geqslant 2 \sqrt{H}-n-2$;

(iii) если $\log H /(\alpha(k) \log \alpha(l)) \rightarrow \infty$, mo

$$
L_{\times}(f) \geqslant(2-o(1))(\alpha(l)-\alpha(k)+1)^{-1 /(2 \alpha(k)-1)} H^{\alpha(k) /(2 \alpha(k)-1)}-n-2 .
$$

Далее, применяя теорему, мы везде за исключением следующего параграфа (об аддитивной сложности перманента) будем вместо $h$ использовать простую оценку $h^{*} \geqslant h$, которая устанавливается следующей леммой. 
Лемма 1. Справедливо соотношение $h \leqslant h^{*}=\max \left\{(k-1)^{3},(l-1)^{2}\right\}$.

ДокАЗАтельство. Покажем, что для любого множества $A=A_{1} \times \cdots \times A_{p} \subset$ mon $f,\left|A_{i}\right|<l$, выполнено $|A| \leqslant h^{*}$.

В случае $p=2$, очевидно, $\left|A_{1} \times A_{2}\right| \leqslant(l-1)^{2}$. При $p=3$ перенумеруем множества $A_{i}$ в порядке неубывания мощности так, что $\left|A_{1}\right| \leqslant\left|A_{2}\right| \leqslant\left|A_{3}\right|$. Если $\left|A_{1} \times A_{2}\right| \geqslant l$, то $\left|A_{3}\right| \leqslant k-1$, откуда следует $\left|A_{1} \times A_{2} \times A_{3}\right| \leqslant\left|A_{3}\right|^{3} \leqslant(k-1)^{3}$. Иначе, если $\left|A_{1} \times A_{2}\right| \leqslant l-1$, то имеем $\left|\left(A_{1} \times A_{2}\right) \times A_{3}\right| \leqslant(l-1)^{2}$.

В общем случае $p>3$ определим $s$ как наибольшее число такое, что $\mid A_{1} \times$ $\cdots \times A_{s} \mid<l$. Если $s=p$, то доказывать нечего. Если $s=p-1$, то получаем $\left|\left(A_{1} \times \cdots \times A_{p-1}\right) \times A_{p}\right| \leqslant(l-1)^{2}$. Иначе положим $A_{1}^{\prime}=A_{1} \times \cdots \times A_{s}, A_{2}^{\prime}=A_{s+1}$, $A_{3}^{\prime}=A_{s+2} \times \cdots \times A_{p}$. Имеем $A_{1} \times \cdots \times A_{p}=A_{1}^{\prime} \times A_{2}^{\prime} \times A_{3}^{\prime}$, причем $\left|A_{1}^{\prime}\right|,\left|A_{2}^{\prime}\right| \leqslant l-1$, $\left|A_{3}^{\prime}\right| \leqslant k-1 \leqslant l-1$. Таким образом, к подмножеству $A_{1}^{\prime} \times A_{2}^{\prime} \times A_{3}^{\prime}$ можно применить рассуждение разобранного выше случая $p=3$. Лемма доказана.

Прежде чем приступить к доказательству теоремы, сделаем несколько замечаний, устанавливающих связь между сформулированной теоремой и результатами работ [39], [40], [29], упоминавшихся во введении.

Пусть в группе $(G,+)$ нет элементов порядка 2 .

Подмножество $H$ группы $(G,+)$ назовем полуразностным, если для любых элементов $a, b, c, d \in H$ справедлива импликация

$$
0 \neq a-b=c-d \quad \Longrightarrow \quad(a=c) \&(b=d) .
$$

Несложно проверить, что в абелевой группе $(G,+)$ подмножество $H$ является полуразностным тогда и только тогда, когда для любых элементов $a, b, c, d \in H$ справедливо

$$
a+b=c+d \quad \Longrightarrow \quad((a=c) \&(b=d)) \vee((a=d) \&(b=c)) .
$$

Такое подмножество еще называется множеством Сидона, см. [52].

В методе [39], [40] в качестве mon $f$ используются полуразностностные подмножества $\{0,1\}^{n} \subset\left(\mathbb{Z}^{n},+\right)$.

Покажем, что в абелевой группе определения полуразностного и 2-редкого подмножества эквивалентны. Пусть $H \subset G$ не является 2-редким, т.е. для некоторых элементов $a \neq b, c \neq d$ выполнено $a+c, a+d, b+c, b+d \in H$. Но тогда $0 \neq(b+c)-(a+c)=(b+d)-(a+d)$, при этом $b+c \neq b+d$, значит, $H$ не является полуразностным.

Пусть теперь $H$ не является полуразностным, т.е. для некоторых элементов $a, b, c, d \in H$ выполняется $a+b=c+d$, при этом $a \neq c, a \neq d$. Но тогда $\{a, d\} \times\{c-a, 0\}=\{a, b, c, d\} \subset H$, т.е. $H$ не является 2-редким.

Поэтому результат [39], [40] вытекает из п. (i) теоремы 1 (при предъявлении 2-редкого подмножества $H \subset\{0,1\}^{n}$ мощности $2^{\lfloor n / 2\rfloor}$, см. далее теорему 3 , п. (i.b)).

В [29] множество $M \subset N^{n}$ называется разделенным, если для любых векторов $r, s, t \in M$ и $u \in N^{n}$ справедлива импликация

$$
r+u=s+t \quad \Longrightarrow \quad(r=s) \vee(r=t) .
$$

Ясно, что разделенное множество является полуразностным, а поэтому и 2редким. Значит, из п. (i) теоремы 1 следуют все результаты п. 4 из [29]. 
ДокАЗАТЕЛЬСтво теоремы 1. От схем, реализующих $f$, перейдем к схемам, реализующим mon $f$ над базисом $\{\mathrm{V}, \times\}$, воспользовавшись следующей леммой. (Обозначаем наименьшее число элементов $\vee$ и элементов $x$, необходимое для реализации множества mon $f$ такими схемами, соответственно через $L_{\vee}(\operatorname{mon} f)$ и $L_{\times}(\operatorname{mon} f)$.)

Лемма 2. Выполняются неравенства

$$
L_{\times}(f) \geqslant L_{\times}(\operatorname{mon} f), \quad L_{+}(f) \geqslant L_{\vee}(\operatorname{mon} f) .
$$

ДокАЗАТЕЛЬство. Пусть $\widetilde{S}$ - произвольная схема, реализующая некоторый многочлен $f$. Заменим в ней элементы умножения элементами $\times$, а элементы сложения - элементами $\vee$. Входы $x_{i}$ заменим константами

$$
a_{i}=\{(\underbrace{0, \ldots, 0,1}_{i}, 0, \ldots, 0)\} \in P\left(N^{n}\right), \quad i=1, \ldots, n,
$$

а константы из $\mathbb{R}_{+}$заменим константой $\{(0, \ldots, 0)\} \in P\left(N^{n}\right)$. Получится схема $S$, вычисляющая множество mon $f$ в полукольце $P\left(N^{n}\right)$ (это следует из гомоморфности отображения mon) и содержащая столько же элементов $\times$ (и соответственно элементов $\vee$ ), сколько в схеме $\widetilde{S}$ было элементов умножения (соответственно элементов сложения). Лемма доказана.

Введем несколько определений и обозначений. Произвольный элемент схемы $S$ будем обозначать символом $e$. Через $\varphi(e)$ обозначим элемент полукольца $P\left(N^{n}\right)$, реализуемый на выходе элемента $e$. Весом элемента $е$ будем называть мощность множества $\varphi(e)$. Без ограничения общности будем считать, что в схеме $S$ нет элементов, не соединенных с выходом хотя бы одной ориентированной цепью (такие элементы можно удалить из схемы). Предками элемента $е$ будем называть элементы, ребра из которых ведут в $e$.

У каждого элемента умножения $\times$ выделим одного из предков и ребро, соединяющее их, назовем запрещенным. Ниже мы покажем, что всегда можно выбрать предка с весом, меньшим $l$. Пусть $\psi(e)$ - элемент полукольца $P\left(N^{n}\right)$, вычисляемый указанным предком элемента $e$. Далее будем рассматривать только (ориентированные) цепи, не проходящие через запрещенные ребра. Пусть $C$ - некоторая цепь, соединяющая элемент $e$ с выходом схемы $S$, и пусть $e_{1}, \ldots, e_{m}$ - все элементы умножения в цепи $C$, не считая $e$ (возможно, что $m=0)$. Положим $\Psi(C)=\psi\left(e_{1}\right) \times \cdots \times \psi\left(e_{m}\right)$, а в случае $m=0$ определим $\Psi(C)=\{(0, \ldots, 0)\}$. Дизъюнкцию $\bigvee_{C} \Psi(C)$, взятую по всем цепям $C$, соединяющим элемент $e$ с выходом схемы $S$, обозначим $\Psi(e)$ (если таких цепей нет, то положим $\Psi(e)=\varnothing)$.

Множество элементов или констант схемы назовем сечением, если любая цепь, соединяющая константу с выходом схемы проходит хотя бы через один элемент из этого множества.

Лемма 3. Пусть Е - произвольное сечение схемъ. Тогда справедливо равенство

$$
\operatorname{mon} f=\bigvee_{e \in E} \varphi(e) \times \Psi(e) .
$$


ДокаЗАТЕльство. Включение mon $f \supset \bigvee_{e \in E} \varphi(e) \times \Psi(e)$ следует из очевидного включения mon $f \supset \varphi(e) \times \Psi(C)$ для любой цепи $C$, соединяющей элемент $e$ с выходом схемы.

Обратное включение mon $f \subset \bigvee_{e \in E} \varphi(e) \times \Psi(e)$ доказывается индукцией по сложности схемы $S$. База индукции, когда схема состоит из одной константы, очевидна.

Докажем индуктивный переход. Пусть элемент $e^{\prime}-$ выход схемы. Будем считать, что $e^{\prime} \notin E$, иначе включение очевидно выполняется.

Рассмотрим случай, когда элемент $e^{\prime}$ является дизъюнкцией. Обозначим через $e_{1}^{\prime}$ и $e_{2}^{\prime}$ его предков и через $S_{i}$ - схему, получаемую из $S$ удалением элемента $e^{\prime}$ и имеющую выходом элемент $e_{i}^{\prime}, i=1,2$. Если $e_{1}^{\prime}=e_{2}^{\prime}$, то доказываемое соотношение следует немедленно, если перейти к рассмотрению подсхемы $S_{1}$. Поэтому далее будем считать, что $e_{1}^{\prime} \neq e_{2}^{\prime}$.

Пусть $C$ - произвольная цепь, соединяющая внутренний элемент схемы $S$ с элементом $e^{\prime}$. Предшествующим ему элементом в цепи $C$ является один из элементов $e_{i}^{\prime}$, причем цепь $C$ может быть получена добавлением элемента $e^{\prime}$ к соответствующей цепи $C^{\prime}$ подсхемы $S_{i}$. Ясно, что $\Psi(C)=\Psi\left(C^{\prime}\right)$.

Положим $E_{i}=E \cap S_{i}$. Для любого элемента $e_{i} \in E_{i}$ и подсхемы $S_{i}$ определим $\Psi_{i}(e)$ аналогично $\Psi(e)$. Тогда $\Psi(e)=\Psi_{i}(e)$ для любого $e \in E_{i} \backslash E_{3-i}$ и $\Psi(e)=$ $\Psi_{1}(e) \vee \Psi_{2}(e)$ для любого $e \in E_{1} \cap E_{2}$. Применяя к подсхемам $S_{i}$ предположение индукции и используя дистрибутивность, получаем нужное включение

$$
\begin{aligned}
\operatorname{mon} f= & \varphi\left(e^{\prime}\right)=\varphi\left(e_{1}^{\prime}\right) \vee \varphi\left(e_{2}^{\prime}\right) \subset\left(\bigvee_{e \in E_{1}} \varphi(e) \times \Psi_{1}(e)\right) \vee\left(\bigvee_{e \in E_{2}} \varphi(e) \times \Psi_{2}(e)\right) \\
= & \left(\bigvee_{e \in E_{1} \backslash E_{2}} \varphi(e) \times \Psi_{1}(e)\right) \vee\left(\bigvee_{e \in E_{2} \backslash E_{1}} \varphi(e) \times \Psi_{2}(e)\right) \\
& \vee\left(\bigvee_{e \in E_{1} \cap E_{2}} \varphi(e) \times\left(\Psi_{1}(e) \vee \Psi_{2}(e)\right)\right)=\bigvee_{e \in E} \varphi(e) \times \Psi(e) .
\end{aligned}
$$

Осталось рассмотреть случай, когда $e^{\prime}$ является элементом умножения. Тогда mon $f=\varphi\left(e^{\prime}\right)=\varphi\left(e_{1}^{\prime}\right) \times \psi\left(e^{\prime}\right)$, где $e_{1}^{\prime}$ - единственный элемент, соединенный с $e^{\prime}$ не запрещенным ребром. Обозначим через $S_{1}$ схему, получаемую из $S$ удалением элемента $e^{\prime}$ и имеющую выходом элемент $e_{1}^{\prime}$.

Любая цепь $C$ в схеме $S$, соединяющая внутренний элемент с элементом $e^{\prime}$, может быть получена добавлением элемента $e^{\prime}$ к некоторой цепи $C^{\prime}$, содержащейся в подсхеме $S_{1}$ и заканчивающейся элементом $e_{1}^{\prime}$. Так как $\Psi(C)=$ $\Psi\left(C^{\prime}\right) \times \psi\left(e^{\prime}\right)$, то, применяя предположение индукции к подсхеме $S_{1}$ и используя дистрибутивность, получаем, что $\Psi(e)=\Psi_{1}(e) \times \psi\left(e^{\prime}\right)$ для любого $e \in E$ (где $\Psi_{1}(e)$ определяется так же, как и в предыдущем случае). Отсюда следует нужное включение

$$
\begin{aligned}
\operatorname{mon} f & =\varphi\left(e^{\prime}\right)=\varphi\left(e_{1}^{\prime}\right) \times \psi\left(e^{\prime}\right) \subset\left(\bigvee_{e \in E} \varphi(e) \times \Psi_{1}(e)\right) \times \psi\left(e^{\prime}\right) \\
& =\bigvee_{e \in E} \varphi(e) \times \Psi_{1}(e) \times \psi\left(e^{\prime}\right)=\bigvee_{e \in E} \varphi(e) \times \Psi(e) .
\end{aligned}
$$

Лемма доказана. 
ЛЕмма 4. Пусть е-элемент умножения схемъ $S$, реализующей $(k, l)$-редкое подмножество mon $f$ множества $N^{n}$. Тогда один из предков элемента е имеет вес, меньший $l$.

ДокАЗАТЕЛЬСтво. Предположим противное. Тогда $\varphi(e)=\varphi_{1} \times \varphi_{2}$, где $\left|\varphi_{i}\right| \geqslant l$. Рассмотрим некоторое сечение схемы, содержащее элемент $e$ (его можно добавить к любому сечению). Применяя к этому сечению предыдущую лемму, получаем включение $\operatorname{mon} f \supset \varphi(e) \times \Psi(e)=\varphi_{1} \times\left(\varphi_{2} \times \Psi(e)\right)$. В силу $\left|\varphi_{2} \times \Psi(e)\right| \geqslant\left|\varphi_{2}\right| \geqslant l$, приходим к противоречию с условием $(k, l)$-редкости множества mon $f$.

При выводе этих соотношений используется ассоциативность умножения и неравенство $|A \times B| \geqslant \max \{|A|,|B|\}$, вытекающее из очевидных включений $\{a\} \times B \subset A \times B$ и $A \times\{b\} \subset A \times B$, где $a \in A$ и $b \in B$ (на самом деле верно более сильное неравенство $|A \times B| \geqslant|A|+|B|-1$ ). Лемма доказана.

Начиная с этого момента, предполагаем, что в схеме $S$ у каждого элемента умножения в качестве запрещенного выбрано ребро, выходящее из предка с весом, меньшим $l$. Тогда, согласно этой договоренности, можно считать, что для любого элемента умножения $e$ справедливо $|\psi(e)|<l$. Иначе можно представить дело так, что каждый элемент умножения $e$ заменен на одновходовый элемент умножения на $\psi(e)$.

В каждой цепи схемы $S$, соединяющей вход и выход схемы, выберем ближайший к входу элемент дизъюнкции, имеющий вес не меньше $l$ (если такой элемент есть) и предшествующий ему элемент (или константу) в этой цепи. Обозначим через $V=\left\{v_{1}, \ldots, v_{m}\right\}$ множество выбранных элементов дизъюнкции, а через $U=\left\{u_{1}, \ldots, u_{t}\right\}-$ множество выбранных их предшественников (возможно, что $m=0$ ).

Случай $m=0$ означает отсутствие в схеме $S$ элементов дизъюнкции с весом, большим $l-1$. Покажем, что в этом случае теорема справедлива. В такой схеме любая цепь, соединяющая константу и выход схемы, заканчивается последовательностью элементов умножения (возможно, пустой), которой предшествует константа или элемент дизъюнкции веса меньше $l$. Значит, схема реализует множество вида $A_{1} \times \cdots \times A_{p}$, где $\left|A_{i}\right|<l$ для всех $i$. Следовательно, $|\operatorname{mon} f| \leqslant h$ (по определению $h$ ), а при этом условии утверждение теоремы справедливо.

С этого момента считаем, что $m>0$.

Лемма 5. Множества $V$ и $U$ являются сечениями схемы $S$.

ДоказАтельство. Докажем, что $V$ - сечение. Предположим, что некоторая цепь $C$ не пересекается с $V$, т.е. она не содержит элементы дизъюнкции веса больше $l-1$. Рассмотрим произвольную цепь $C^{\prime}$, пересекающуюся с $V$. Цепи $C$ и $C^{\prime}$ имеют общий элемент $e^{\prime}$ - выход схемы, таким образом, они имеют некоторую общую подцепь $C^{\prime \prime}$, которая заканчивается элементом $e^{\prime}$. Пусть $e-$ первый элемент этой подцепи, считая от входов.

Элементы множества $V \cap C^{\prime}$ не содержатся в подцепи $C^{\prime \prime}$, следовательно, они предшествуют элементу $e$ в цепи $C^{\prime}$, откуда следует, что $e$ имеет вес не меньше $l$. Так как $e \in C$, то $e$ не может быть элементом дизъюнкции. Тогда $e-$ элемент умножения, но элемент умножения является одновходовым, тогда его 
предок также принадлежит $C \cap C^{\prime}$, что противоречит выбору $e$ как первого элемента в общей подцепи $C$ и $C^{\prime}$.

Выше показано, что в любой цепи схемы имеется элемент из множества $V$. Следовательно, по построению в ней также имеется элемент (или константа) из множества $U$. Лемма доказана.

Выполним следующее преобразование схемы $S$. Заменим каждый элемент $u_{i}$ на соответствующую константу, реализующую $\varphi\left(u_{i}\right)$, эту константу также обозначим $u_{i}$. Удалим из схемы все ребра, ведущие в элементы $u_{i}$; если все ребра, выходящие из некоторого элемента, будут удалены, то удалим сам этот элемент вместе со всеми входящими в него ребрами и т.д.

В результате указанных преобразований множество, реализуемое схемой, не изменяется, а число элементов дизъюнкции, а также число остальных элементов не увеличивается. Новую схему будем обозначать $\widehat{S}$.

Определим матрицу $\left(\beta_{i, j}\right)$ следующим образом: положим $\beta_{i, j}=1$ в том и только том случае, если ребро $\left(u_{i}, v_{j}\right)$ имеется в $\widehat{S}$, и $\beta_{i, j}=0$ иначе.

Лемма 6. Справедливо соотношение

$$
\operatorname{mon} f=\bigvee_{\substack{i, j \\ \beta_{i, j}=1}} \varphi\left(u_{i}\right) \times \Psi\left(v_{j}\right) .
$$

ДокАЗАТЕЛЬство. Из леммы 5 вытекает, что множество $U \cap \widehat{S}$ является сечением схемы $\widehat{S}$. Применяя лемму 3 и используя определение функции $\Psi(e)$ применительно к схеме $\widehat{S}$, получаем

$$
\operatorname{mon} f=\bigvee_{i=1}^{t} \varphi\left(u_{i}\right) \times \Psi\left(u_{i}\right)=\bigvee_{\substack{i, C \\ u_{i} \in C}} \varphi\left(u_{i}\right) \times \Psi(C)
$$

где $\{C\}$ - цепи, соединяющие выходы констант $u_{j}$ с выходом схемы $\widehat{S}$ (если $u_{i} \notin \widehat{S}$, полагаем $\left.\Psi\left(u_{i}\right)=\varnothing\right)$. Пусть $C$ - произвольная такая цепь с началом в константе $u_{i}$.

Покажем, что следующий после $u_{i}$ элемент в этой цепи принадлежит $V$. Во-первых, докажем, что $V \cap \widehat{S}$ - сечение схемы $\widehat{S}$, т.е. в цепи $C$ найдется элемент из $V$, не равный $u_{i}$. Предположим противное. Из определения $u_{i}$ следует, что в схеме $S$ имеется подцепь $C^{\prime \prime}$, соединяющая некоторый вход схемы с элементом $u_{i}$ и не содержащая элементов из $V$. Рассмотрим цепь $C^{\prime}$ в схеме $S$, которая получается продолжением подцепи $C^{\prime \prime}$ цепью $C$. Из определения множества $V$ следует, что $C^{\prime} \cap V \neq \varnothing$. Но в таком случае $\left(C \backslash\left\{u_{i}\right\}\right) \cap V \neq \varnothing$, что противоречит предположению. Значит, $V \cap \widehat{S}$ - сечение схемы $\widehat{S}$.

Если элемент из $V$ не является следующим за $u_{i}$ в цепи $C$, то это означает, что $u_{i}$ предшествует некоторому элементу $u_{j}$, а это противоречит тому, что в схеме $\widehat{S}$ все элементы из $U$ являются константами. Таким образом, все ребра из $U \cap \widehat{S}$ ведут в $V$.

Пусть $u_{i}$ и $v_{j}$ - первые элементы цепи $C$. Остальную часть цепи обозначим через $C^{\prime}$. Тогда $\Psi(C)=\Psi\left(C^{\prime}\right)$ и из того, что множество $\Psi\left(v_{j}\right)$ есть дизъюнкция 
всевозможных $\Psi\left(C^{\prime}\right), v_{j} \in C^{\prime}$, вытекает равенство

$$
\operatorname{mon} f=\bigvee_{\substack{i, C \\ u_{i} \in C}} \varphi\left(u_{i}\right) \times \Psi(C)=\bigvee_{\substack{i, C^{\prime} \\\left\{u_{i}\right\} \cup C^{\prime}-\text { цепь }}} \varphi\left(u_{i}\right) \times \Psi\left(C^{\prime}\right)=\bigvee_{\substack{i, j \\ \beta_{i, j}=1}} \varphi\left(u_{i}\right) \times \Psi\left(v_{j}\right)
$$

Лемма доказана.

Лемма 7. Справедливо соотношение $\sum_{i, j} \beta_{i, j} \geqslant h^{-1}|\operatorname{mon} f|$.

ДокАЗАТЕЛЬСТво. Из того, что

$$
\left|\varphi\left(v_{j}\right)\right| \geqslant l, \quad \varphi\left(v_{j}\right) \times \Psi\left(v_{j}\right) \subset \operatorname{mon} f,
$$

следует, что $\left|\Psi\left(v_{j}\right)\right|<k$ для всех $j$. В противном случае множество $\operatorname{mon} f$ не было бы $(k, l)$-редким.

Проверим теперь, что любое множество $\varphi\left(u_{i}\right)$, где $u_{i} \in U$, можно представить в виде $B_{1} \times \cdots \times B_{p}$, где $\left|B_{j}\right|<l, B_{j} \subset N^{n}$ при любом $j$. По определению множества $U$, существует цепь, в которой элементу (или константе) $u_{i}$ не предшествуют элементы дизъюнкции веса больше $l-1$. Тогда множество $\varphi\left(u_{i}\right)$, вычисляемое подцепью, заканчивающейся $u_{i}$, очевидно имеет требуемый вид.

Из только что доказанных утверждений и леммы 1 получаем, что $\mid \varphi\left(u_{i}\right) \times$ $\Psi\left(v_{j}\right) \mid \leqslant h$, после чего доказываемое неравенство становится прямым следствием леммы 6. Лемма доказана.

Перейдем опять к рассмотрению схемы $\widehat{S}$. Докажем, что число элементов дизъюнкции в ней не меньше чем $\sum_{i, j} \beta_{i, j}-1$.

Обозначим через $r_{i}, i=1,2$, число элементов схемы, в которые входят ровно $i$ ребер, и подсчитаем двумя способами число всех ее ребер. С одной стороны, оно равно $r_{1}+2 r_{2}$ (по числу входов), а с другой стороны, оно не меньше чем $r_{1}+r_{2}+\sum_{i, j} \beta_{i, j}-1$ (по числу выходов). Значит, $r_{2} \geqslant \sum_{i, j} \beta_{i, j}-1$ (по существу, это повторение известных рассуждений, имеющихся, например, в [2]). Окончательно получаем

$$
\sum_{i, j} \beta_{i, j}-1 \leqslant r_{2} \leqslant L_{\vee}(\widehat{S}) \leqslant L_{\vee}(S)
$$

что совместно с леммами 2 и 7 доказывает неравенство (i) теоремы.

Для доказательства утверждения (ii) теоремы в каждой цепи схемы $S$ выберем ближайший к входам элемент $v_{i} \in V$. Также выберем ближайшие к нему предшествующий одновходовый элемент в данной цепи (или константу, с которой начинается цепь, если $v_{i}$ не имеет одновходовых предшественников) и последующий одновходовый элемент (или выход схемы, если $v_{i}$ не имеет одновходовых потомков). Множество всех выбранных предшественников обозначим через $W=\left\{w_{1}, \ldots, w_{p^{\prime}}\right\}$, а множество выбранных потомков - через $Z=\left\{z_{1}, \ldots, z_{q^{\prime}}\right\}$. При этом если выход $e^{\prime}$ схемы $S$ содержится в $Z$, то положим $\psi\left(e^{\prime}\right)=\{(0, \ldots, 0)\}$.

Превратим все элементы из $W$ в константы, рассуждая так же, как при построении схемы $\widehat{S}$. Новую схему обозначим $\breve{S}$. 
Лемма 8. Существует такая булева матрица $\left(\mu_{i, j}\right)$, что

$$
\operatorname{mon} f=\bigvee_{\substack{i, j \\ \mu_{i, j}=1}} \varphi\left(w_{i}\right) \times \psi\left(z_{j}\right) \times \Psi\left(z_{j}\right)
$$

При этом $\mu_{i, j}=0$, если $w_{i} \notin \breve{S}$ или $z_{j} \notin \breve{S}$.

ДокАЗАТЕЛЬСтво. Множество $W \cap \breve{S}$ является сечением схемы $\breve{S}$, поскольку содержит все входы. Поэтому из леммы 3 следует, что

$$
\operatorname{mon} f=\bigvee_{\substack{i, C \\ w_{i} \in C}} \varphi\left(w_{i}\right) \times \Psi(C),
$$

где $\{C\}$ - цепи, соединяющие константы $w_{i}$ с выходом схемы $\breve{S}$. Множества $V \cap \breve{S}$ и $Z \cap \breve{S}$ тоже являются сечениями схемы $\breve{S}$ - это доказывается так же, как в лемме 6 .

Покажем, что $W \cap Z \cap \breve{S}=\varnothing$. Предположим противное, т.е. существует элемент $w_{i}=z_{j} \in \breve{S}$. Очевидно, такой элемент не может быть константой схемы $S$ и не может быть элементом дизъюнкции. Тогда он одновходовый, и в любой цепи, соединяющей вход и выход схемы $S$, ему предшествует один и тот же элемент $e$. Так как в некоторой цепи элементу $z_{j}$ предшествует элемент дизъюнкции с весом не меньше $l$, то $|\varphi(e)| \geqslant l$. Тогда, если $e-$ элемент умножения, то $z_{j}$ не может принадлежать $Z$, а если $e$ - элемент дизъюнкции, то $w_{i}$ не может принадлежать $W$, что противоречит допущению.

Из приведенного рассуждения также вытекает, что предком элемента умножения из множества $Z$ обязательно является элемент дизъюнкции с весом не меньше $l$.

Рассмотрим произвольную цепь $C$ с началом в константе $w_{i}$. По построению схемы $\breve{S}$, в цепи $C$ нет других элементов из $W$, кроме $w_{i}$. Пусть $z_{j}$-ближайший к входу элемент из множества $Z$ в цепи $C$. Покажем, что на участке $C^{\prime \prime}$ цепи между $w_{i}$ и $z_{j}$ нет других одновходовых элементов.

Во-первых, на этом участке обязательно есть элемент дизъюнкции с весом не меньше $l$. Если элемент $z_{j}$ сам не является элементом дизъюнкции, т.е. выходом схемы, то это следует из приведенного выше замечания о предке одновходового элемента из $Z$. Пусть $v-$ ближайший к $w_{i}$ такой элемент дизъюнкции. Тогда, согласно определению $w_{i}$, элемент $v$ является ближайшим к входу элементом дизъюнкции с весом не меньше $l$ в некоторой цепи схемы $S$, содержащей подцепь $C^{\prime \prime}$, т.е. $v \in V$. Тогда, если допустить существование отличного от $w_{i}$ и $z_{j}$ одновходового элемента в $C^{\prime \prime}$, из этого автоматически будет следовать существование либо отличного от $w_{i}$ элемента из $W$, либо отличного от $z_{j}$ элемента из $Z$ в рассматриваемой подцепи $C^{\prime \prime}$, что противоречит либо определению схемы $\breve{S}$, либо условию выбора элемента $z_{j}$ соответственно.

Таким образом, между элементами $w_{i}$ и $z_{j}$ могут быть только элементы дизъюнкции.

Рассмотрим подцепь $C^{\prime}$ цепи $C$, соединяющую элемент $z_{j}$ с выходом схемы. Согласно определению функции $\Psi$, справедливо $\Psi(C)=\psi\left(z_{j}\right) \times \Psi\left(C^{\prime}\right)$. Как следствие, любую цепь $C^{\prime}$, соединяющую элемент $z_{j}$ с выходом схемы, можно 
удлинить до некоторой цепи $C$, соединяющей элемент $w_{i}$ с выходом схемы и такой, что $\Psi(C)=\psi\left(z_{j}\right) \times \Psi\left(C^{\prime}\right)$. Поэтому

$$
\psi\left(z_{j}\right) \times \Psi\left(z_{j}\right)=\bigvee_{C^{\prime}} \psi\left(z_{j}\right) \times \Psi\left(C^{\prime}\right)=\bigvee_{\substack{C \\ w_{i}, z_{j} \in C}} \Psi(C),
$$

где первая дизъюнкция берется по подцепям $\left\{C^{\prime}\right\}$ с началом в $z_{j}$. Отсюда вытекает существование булевой матрицы $\left(\mu_{i, j}\right)$ такой, что

$$
\operatorname{mon} f=\bigvee_{\substack{i, C \\ w_{i} \in C}} \varphi\left(w_{i}\right) \times \Psi(C)=\bigvee_{\substack{i, j \\ \mu_{i, j}=1}} \varphi\left(w_{i}\right) \times \psi\left(z_{j}\right) \times \Psi\left(z_{j}\right)
$$

При этом по построению в последней дизъюнкции учитываются только пары элементов $w_{i}$ и $z_{j}$ схемы $\breve{S}$. Лемма доказана.

Лемма 9. Справедливо соотношение $\sum_{i, j} \mu_{i, j} \geqslant h^{-1}|\operatorname{mon} f|$.

ДокАЗАтЕльство. Как и при доказательстве леммы 7 , достаточно показать, что любое множество $\varphi\left(w_{i}\right) \times \psi\left(z_{j}\right) \times \Psi\left(z_{j}\right)$ представимо в виде произведения множеств, каждое из которых имеет вес меньше $l$. После этого требуемое неравенство будет прямым следствием лемм 1 и 8.

Ясно, что $\left|\psi\left(z_{j}\right)\right|<l$. Кроме того, так как $\left|\varphi\left(z_{j}\right)\right| \geqslant l$ и $\varphi\left(z_{j}\right) \times \Psi\left(z_{j}\right) \subset \operatorname{mon} f$, то $\left|\Psi\left(z_{j}\right)\right|<k \leqslant l$. Наконец, множество $\varphi\left(w_{i}\right)$ вычисляется некоторой цепью, в которой нет элементов дизъюнкции веса больше $l-1$. Значит, множество $\varphi\left(w_{i}\right)$ и, как следствие, множество $\varphi\left(w_{i}\right) \times \psi\left(z_{j}\right) \times \Psi\left(z_{j}\right)$ имеет необходимый вид. Лемма доказана.

Пусть $|W \cap \breve{S}|=p$ и $|Z \cap \breve{S}|=q$. Тогда, поскольку все элементы из $W \cup Z$, за исключением, быть может, $n+2$ элементов, являются одновходовыми, и поскольку $W \cap Z \cap \breve{S}=\varnothing$ (доказано в лемме 8), то

$$
L_{\times}(\breve{S}) \geqslant p+q-n-2 .
$$

Используя неравенства $p+q \geqslant 2 \sqrt{p q}$ и $p q \geqslant \sum_{i, j} \mu_{i, j}$, получаем

$$
L_{\times}(S) \geqslant L_{\times}(\breve{S}) \geqslant p+q-n-2 \geqslant 2 \sqrt{\sum_{i, j} \mu_{i, j}}-n-2,
$$

что вместе с леммами 2 и 9 доказывает неравенство (ii) теоремы.

Докажем неравенство (iii), которое уточняет (ii) в асимптотическом смысле. Для удобства рассуждений удалим из множеств $W$ и $Z$ элементы, которые отвечают нулевым строкам и соответственно нулевым столбцам матрицы $\left(\mu_{i, j}\right)$. Удалим нулевые строки и столбцы из матрицы $\left(\mu_{i, j}\right)$ - новую матрицу будем далее также обозначать $\left(\mu_{i, j}\right)$. Утверждение леммы 8 останется в силе для таких множеств (при подходящей переиндексации элементов) и такой матрицы.

Теперь предположим, что матрица $\left(\mu_{i, j}\right)$ имеет наименьшие размеры (число строк и число столбцов) среди всех, для которых справедлива лемма 8.

Тогда ни одно из множеств $\varphi\left(w_{i}\right)$ не является дизъюнкцией других множеств $\varphi\left(w_{j}\right), j \neq i$, и аналогичное утверждение справедливо в отношении множеств 
$\psi\left(z_{i}\right) \times \Psi\left(z_{i}\right)$. Действительно, если бы, например, множество $\varphi\left(w_{i}\right)$ представлялось бы в виде $\bigvee_{j \in J} \varphi\left(w_{j}\right), i \notin J$, то заменив в формуле из утверждения леммы 8 все вхождения $\varphi\left(w_{i}\right)$ на $\bigvee_{j \in J} \varphi\left(w_{j}\right)$, выполнив соответствующие преобразования и удалив $w_{i}$ из множества $W$, получили бы аналогичную формулу с новой матрицей $\left(\mu_{i, j}^{\prime}\right)$ с меньшим числом строк.

Будем называть булеву матрицу $(k, l)$-редкой, если она не содержит $(k \times l)$ подматрицу, все элементы которой - единицы.

Лемма 10. Матрица $\left(\mu_{i, j}\right)$ является $(\alpha(k), \alpha(l))$-редкой и $(\alpha(l), \alpha(k))$-редкой.

ДокАзАТЕльство. Предположим, например, что матрица $\left(\mu_{i, j}\right)$ не является $(\alpha(k), \alpha(l))$-редкой. Тогда для некоторых $\alpha(k)$-элементного множества $I$ и $\alpha(l)$-элементного множества $J$ согласно лемме 8 справедливо включение

$$
\left(\bigvee_{i \in I} \varphi\left(w_{i}\right)\right) \times\left(\bigvee_{j \in J} \psi\left(z_{j}\right) \times \psi\left(Z_{j}\right)\right) \subset \operatorname{mon} f
$$

Если допустить, что $\left|\bigvee_{i \in I} \varphi\left(w_{i}\right)\right|<k$, то в некотором $(k-1)$-элементном множестве найдется система из $\alpha(k)$ различных непустых подмножеств, ни одно из которых не является дизъюнкцией двух других (и даже любого числа других). Добавляя пустое подмножество, получаем систему из $\alpha(k)+1$ подмножеств с тем же свойством, а это противоречит определению величины $\alpha(k)$. Поэтому $\left|\bigvee_{i \in I} \varphi\left(w_{i}\right)\right| \geqslant k$ и аналогично $\left|\bigvee_{j \in J} \psi\left(z_{j}\right) \times \psi\left(Z_{j}\right)\right| \geqslant l$. Но это противоречит $(k, l)$-редкости множества mon $f$.

Аналогично проверяется то, что матрица $\left(\mu_{i, j}\right)$ является $(\alpha(l), \alpha(k))$-редкой. Лемма доказана.

Следующая лемма вытекает непосредственно из оценки веса редкой булевой матрицы из работы [53] (в этой работе уточняется соответствующая оценка [54]). Число единиц в булевой матрице далее называем ее весом.

Лемма 11. Пусть $\alpha \leqslant \beta$. Тогда вес $(\alpha, \beta)$-редкой $и(\beta, \alpha)$-редкой булевой $(p \times q)$-матрици $A$ не превосходит

$$
(\beta-\alpha+1)^{1 / \alpha}\left(\frac{p+q}{2}\right)^{2-1 / \alpha}+(\alpha-1)\left(\frac{p+q}{2}\right)^{2-2 / \alpha}+(\alpha-2) \frac{p+q}{2} .
$$

ДоказАтЕльство. Верхняя оценка [53] веса $(\beta, \alpha)$-редкой $(p \times q)$-матрицы $A$ имеет вид

$$
(\beta-\alpha+1)^{1 / \alpha} p^{1-1 / \alpha} q+(\alpha-1) p^{2-2 / \alpha}+(\alpha-2) p .
$$

Выпишем аналогичную оценку для веса транспонированной матрицы (она также $(\beta, \alpha)$-редкая) и возьмем арифметическое среднее двух оценок. Используя известные соотношения

$$
p q \leqslant\left(\frac{p+q}{2}\right)^{2}, \quad \frac{p^{1 / \alpha}+q^{1 / \alpha}}{2} \leqslant\left(\frac{p+q}{2}\right)^{1 / \alpha}
$$


из которых следует

$$
\begin{aligned}
\frac{p^{1-1 / \alpha} q+q^{1-1 / \alpha} p}{2} & =\frac{p q}{2}\left(\frac{1}{p^{1 / \alpha}}+\frac{1}{q^{1 / \alpha}}\right) \leqslant \frac{p q}{2}\left(\frac{1}{p}+\frac{1}{q}\right)^{1 / \alpha} \\
& =(p q)^{1-1 / \alpha}\left(\frac{p+q}{2}\right)^{1 / \alpha} \leqslant\left(\frac{p+q}{2}\right)^{2-1 / \alpha}
\end{aligned}
$$

в итоге получаем утверждение леммы. Лемма доказана.

Из леммы 9 и двух последних лемм вытекает оценка

$$
\begin{aligned}
& h^{-1}|\operatorname{mon} f| \leqslant( \\
& \quad(l)-\alpha(k)+1)^{1 / \alpha(k)}\left(\frac{p+q}{2}\right)^{2-1 / \alpha(k)} \\
& \quad+(\alpha(k)-1)\left(\frac{p+q}{2}\right)^{2-2 / \alpha(k)}+(\alpha(k)-2) \frac{p+q}{2},
\end{aligned}
$$

из которой следует, что

$$
p+q \geqslant 2\left(D-a D^{(2 \alpha(k)-2) /(2 \alpha(k)-1)}-b D^{\alpha(k) /(2 \alpha(k)-1)}\right)^{\alpha(k) /(2 \alpha(k)-1)},
$$

где

$$
\begin{gathered}
D=\frac{|\operatorname{mon} f|}{h(\alpha(l)-\alpha(k)+1)^{1 / \alpha(k)}}, \\
a=\frac{\alpha(k)-1}{(\alpha(l)-\alpha(k)+1)^{1 / \alpha(k)}}, \quad b=\frac{\alpha(k)-2}{(\alpha(l)-\alpha(k)+1)^{1 / \alpha(k)}},
\end{gathered}
$$

если выражение внутри скобок (1) положительно, а это гарантируется условием п. (iii): можно считать, что при достаточно большом $D$ выполнено $D>(a+b) \times$ $D^{(2 \alpha(k)-2) /(2 \alpha(k)-1)}$. Теперь неравенство (iii) следует из того, что $L_{\times}(f) \geqslant p+$ $q-n-2$. Теорема доказана.

ЗАмечАниЕ 1. Что также следует из доказательства, в неравенстве (iii) функцию $\alpha(k)$ можно заменить функцией $\alpha^{*}(k)$, определение которой отличается от определения $\alpha(k)$ тем, что запрещаются дизъюнкции не только двух, но и любого числа других векторов. Впрочем, это не приводит к существенному уточнению оценок, поскольку, как следует из результата Клейтмена, $\alpha^{*}(k)$ $\alpha(k)$, кроме того, $\alpha^{*}(k)=\alpha(k)$ при $k \leqslant 4$.

ЗАмечание 2. В частном случае $k=2$ из п. (iii) следует оценка $L_{\times}(f)=$ $\Omega_{l}\left(|\operatorname{mon} f|^{2 / 3}\right)$, а в случае $k=3$ - оценка $L_{\times}(f)=\Omega_{l}\left(|\operatorname{mon} f|^{3 / 5}\right)$. Далее в теореме 6 будет показано, что эти оценки по порядку неулучшаемы.

\section{§ 4. Аддитивная сложность перманента}

Обозначим

$$
\operatorname{per}_{n}=\sum_{\pi \in S_{n}} x_{1, \pi(1)} \cdots x_{n, \pi(n)},
$$

где $S_{n}-$ множество всех перестановок $\{1, \ldots, n\}$. 
Лемма 12. Пусть $f=\operatorname{per}_{n}$. Тогда при любом $m=0, \ldots,\lceil n / 2\rceil-1$ множество mon $f$ является $(m !+1,(n-m-1) !+1)$-редким.

Доказательство. Пусть $A \times B \subset \operatorname{mon} f$. Обозначим через $R_{A}$ множество номеров всех ненулевых строк матриц из $A$, а через $C_{A}-$ множество номеров ненулевых столбцов. Через $R_{B}$ и $C_{B}$ обозначим аналогичные множества строк и столбцов матриц из $B$.

Ясно, что компонентами матрицы из $A \cup B$ могут быть только нули и единицы. Также ясно, что в любой строке или столбце такой матрицы может быть не более одной единицы. Поэтому в каждой матрице из $A$ (соответственно из $B$ ) не более $\min \left\{\left|R_{A}\right|,\left|C_{A}\right|\right\}$ (соответственно $\left.\min \left\{\left|R_{B}\right|,\left|C_{B}\right|\right\}\right)$ единиц.

Проверим, что $R_{A} \cap R_{B}=\varnothing=C_{A} \cap C_{B}$. Действительно, если, например, $R_{A} \cap$ $R_{B} \neq \varnothing$, то в множествах $A$ и $B$ есть матрицы, имеющие единицы в одинаковых строках, тогда сумма этих матриц в соответствующей строке содержит либо двойку, либо больше одной единицы, значит, эта сумма не принадлежит mon $f$.

Теперь, замечая, что общее число единиц в любых двух матрицах из $A$ и $B$ равно $n$, получаем $\left|R_{A}\right|+\left|R_{B}\right|=\left|C_{A}\right|+\left|C_{B}\right|=n$, при этом $\left|R_{A}\right|=\left|C_{A}\right|=k$ и $\left|R_{B}\right|=\left|C_{B}\right|=n-k$. Следовательно, в каждой матрице из $A$ содержится $k$ единиц, расположенных по одной в каждой строке из $R_{A}$ и каждом столбце из $C_{A}$. Поэтому $|A| \leqslant k$ !. Аналогично получаем, что $|B| \leqslant(n-k) !$.

Следовательно, при любом $m=0, \ldots, n$ не может одновременно выполняться $|A|>m !$ и $|B|>(n-1-m)$ !. Лемма доказана.

Теорема 2. Для аддитивной сложности перманента справедливо неравенство

$$
L_{+}\left(\operatorname{per}_{n}\right) \geqslant \frac{n !}{(\lfloor n / 2\rfloor !)^{2}}-1 .
$$

ДокАЗАТЕЛЬство. Покажем, что для величины $h$ из формулировки теоремы 1 справедливо $h \leqslant(\lfloor n / 2\rfloor !)^{2}$.

Согласно лемме 12 в определении $h$ можно положить $l=\lfloor n / 2\rfloor !+1$. Пусть $A_{1} \times \cdots \times A_{p} \subset \operatorname{mon} f,\left|A_{i}\right| \leqslant\lfloor n / 2\rfloor$ ! для всех $i=1, \ldots, p$, любое множество $A_{i}$ состоит из булевых матриц размера $n \times n$.

Повторяя рассуждения из доказательства леммы 12 , получаем, что множество $A_{i}$ состоит из булевых матриц, каждая из которых содержит ровно $k_{i}$ единиц, которые расположены по одной в каждой из строк с номерами из множества $R_{i}$ и в каждом из столбцов с номерами из множества $C_{i}$, при этом

$$
\left|R_{i}\right|=\left|C_{i}\right|=k_{i}, \quad \sum_{i=1}^{p} k_{i}=n, \quad \bigcup_{i=1}^{p} R_{i}=\bigcup_{i=1}^{p} C_{i}=\{1, \ldots, n\}, \quad\left|A_{i}\right| \leqslant k_{i} ! .
$$

Без ограничения общности можно считать, что $k_{1} \geqslant k_{i} \geqslant 1$ для всех $i$. Так как при $p=2$ неравенство $\left|A_{1} \times \cdots \times A_{p}\right| \leqslant(\lfloor n / 2\rfloor !)^{2}$ очевидно, далее считаем, что $p \geqslant 3$.

Если $k_{1} \geqslant\lfloor n / 2\rfloor$, то

$$
\left|A_{1} \times \cdots \times A_{p}\right| \leqslant\left|A_{1}\right| \cdot k_{2} ! \cdots k_{p} ! \leqslant\left\lfloor\frac{n}{2}\right\rfloor !\left(n-k_{1}-1\right) ! \leqslant\left(\left\lfloor\frac{n}{2}\right\rfloor !\right)^{2} .
$$


При выводе используется справедливое при $0<k<n$ неравенство $k !(n-k)$ ! $\leqslant$ $(n-1)$ !, из которого следует $k_{2} ! \cdots k_{p} ! \leqslant\left(k_{2}+\cdots+k_{p}-1\right) !=\left(n-k_{1}-1\right)$ !.

Если $k_{1}<\lfloor n / 2\rfloor$, то, применяя справедливое при $x \leqslant y$ неравенство $x ! y ! \leqslant$ $(x-1) !(y+1)$ !, получаем

$$
\left|A_{1} \times \cdots \times A_{p}\right| \leqslant k_{1} ! \cdots k_{p} ! \leqslant\left\lfloor\frac{n}{2}\right\rfloor ! \cdot k_{2}^{\prime} ! \cdots k_{s}^{\prime} !,
$$

где $1 \leqslant k_{i}^{\prime} \leqslant\lfloor n / 2\rfloor, s \leqslant p, \sum_{i=1}^{s} k_{i}^{\prime}=\lceil n / 2\rceil$, откуда также получаем требуемую оценку. Теорема доказана.

Таким образом, при нечетном $n$ выполняется $L_{+}\left(\operatorname{per}_{n}\right) \geqslant n C_{n-1}^{(n-1) / 2}-1 \asymp$ $\sqrt{n} 2^{n}$. Так как сложность перманента порядка $n$ очевидно не меньше, чем сложность перманента порядка $n-1$, при четном $n$ получаем такую же по порядку оценку. Как известно (см., например, [14; упражнения к п. 4.6.4]), $L_{+}\left(\operatorname{per}_{n}\right)=O\left(n 2^{n}\right)$. Значит, полученная оценка близка к точной.

В то же время оценка для $L_{\times}\left(\operatorname{per}_{n}\right)$, получаемая методом теоремы 1 , существенно уступает истинной величине $L_{\times}\left(\right.$per $\left._{n}\right) \sim n 2^{n-1}$, найденной в [30].

Как следствие, для аддитивной сложности гамильтониана $H C_{n}$, ввиду того, что mon $H C_{n} \subset \operatorname{mon}_{p_{n}}$ и $\left|\operatorname{mon} H C_{n}\right|=(n-1)$ !, имеем оценку $L_{+}\left(H C_{n}\right)=$ $\Omega\left(2^{n} / \sqrt{n}\right)$.

\section{§5. Примеры редких множеств}

Для того чтобы указать способы применения теоремы 1 для получения высоких нижних оценок монотонной сложности индивидуальных многочленов, нам понадобятся эффективные примеры редких множеств достаточно большой мощности. Обозначим $E_{n}=\{0, \ldots, n-1\}$.

Выше отмечалось, что в абелевой группе 2-редкое подмножество - это то же самое, что множество Сидона.

Обзор известных конструкций множеств Сидона, а также оценок мощности таких множеств приводится в [52] и в работах из библиографического указателя к [52]. Для нас важно отметить следующие факты: мощность множества Сидона в $E_{n}$ и в группе $\mathbb{Z}_{n}$ асимптотически не превосходит $\sqrt{n}$. Эта оценка достигается в первом случае при всех (достаточно больших) $n$, а во втором при некоторых $n$. Перечислим наиболее известные примеры.

а) Множество Дж. Зингера мощности $q+1$ в $\mathbb{Z}_{q^{2}+q+1}$, где $q-$ степень простого числа, определяется как

$$
\{0\} \cup\left\{s_{i} \mid \theta^{s_{i}} /\left(\theta+\alpha_{i}\right) \in G F(q), G F(q)=\left\{\alpha_{1}, \ldots, \alpha_{q}\right\}\right\},
$$

где $\theta$ - примитивный элемент в поле $G F\left(q^{3}\right)$. Более подробно см. в [55].

б) Множество Р. Боуза мощности $q$ в $\mathbb{Z}_{q^{2}-1}$, где $q$ - степень простого числа, определяется как $\left\{s_{i} \mid \theta^{s_{i}}=\theta+\alpha_{i}, G F(q)=\left\{\alpha_{1}, \ldots, \alpha_{q}\right\}\right\}$, где $\theta$ - примитивный элемент в поле $G F\left(q^{2}\right)$.

в) Множество В.Е. Алексеева [56] мощности $p-1$ в $\mathbb{Z}_{p(p-1)}$, где $p$ - простое число, определяется как $\left\{s_{i} \mid s_{i} \equiv i \bmod (p-1), s_{i} \equiv \zeta^{i} \bmod p\right\}$, где $\zeta-$ порождающий элемент мультипликативной группы поля $G F(p)$. 
Любой из перечисленных примеров трансформируется в множество Сидона в $E_{n}$ мощности $(1-o(1)) \sqrt{n}$. Это устанавливается, во-первых, тем, что редкое подмножество в $\mathbb{Z}_{Q}$ тем более является редким в $E_{Q}$ (в смысле естественного взаимно однозначного гомоморфизма из $E_{Q}$ в $\left.\mathbb{Z}_{Q}\right)$. Во-вторых, если $Q_{R}(m)-$ ближайшее снизу к $m$ число вида $R(p)$, где $R$ - (фиксированный) многочлен, а $p$ - простое число, то, как известно, $Q_{R}(m) \sim m$ при $m \rightarrow \infty$ (это следует из результатов о плотности распределения простых чисел, см., например, [57]).

Еще несколько примеров редких множеств приводятся в следующей теореме.

Обозначим через $\psi_{n}$ следующее (достаточно естественное) отображение из $G F\left(2^{n}\right)$ в $N^{n}$ (через $G F(q)$ обозначается поле Галуа порядка $q$ ). Пусть используется представление поля $G F\left(2^{n}\right)$ как векторного пространства размерности $n$ над $G F(2)$. Тогда $\psi_{n}$ переводит векторы над $G F(2)$ в векторы над $N$, при этом единичные компоненты - в единицы, нулевые компоненты - в нули.

Tеорема 3. (i.a) B групnе $\left(G F(q)^{2},+\right)$, әде q нечетно, парабола $\left\{\left(x, x^{2}\right) \mid\right.$ $x \in G F(q)\}$ является 2-редким подмножеством мощности $q$.

(i.b) B полугруппе $\left(N^{2 n},+\right)$ “кубическая парабола" $\left\{\left(\psi_{n}(x), \psi_{n}\left(x^{3}\right)\right) \mid x \in\right.$ $\left.G F\left(2^{n}\right)\right\}$ является 2-редким подмножеством мощности $2^{n}$.

(ii.a) $B$ группе $\left(G F(q)^{3},+\right)$, где q нечетно, сфера $\left\{(x, y, z) \mid x^{2}+y^{2}+z^{2}=\gamma\right\}$,

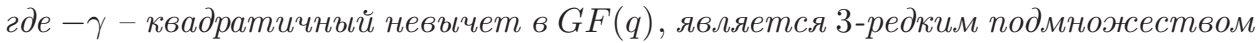
мощности $q^{2}-q$.

(ii.b) B группе $\left(G F(q)^{3},+\right)$, где $q=2^{2 k+1}$, поверхность $\left\{(x, y, z) \mid x^{3}+y^{7}+\right.$ $\left.z^{15}=1\right\}$ является $(47,315)$-редким подмножеством мощности $q^{2}$.

(iii) B группе $\left(G F\left(q^{t}\right),+\right)$ множество $\left\{x \mid x^{\left(q^{t}-1\right) /(q-1)}=1\right\}$ элементов единичной нормы является $(t, t !+1)$-редким подмножеством мощности $\left(q^{t}-\right.$ 1) $/(q-1)$.

ДокАзАтельство. Докажем (i.а). Проверим, что из равенства двумерных векторов над $G F(q)$

$$
\left(x, x^{2}\right)-\left(y, y^{2}\right)=\left(z, z^{2}\right)-\left(u, u^{2}\right) \neq(0,0)
$$

следует, что $x=z$ и $y=u$. Действительно, система уравнений

$$
\left\{\begin{array}{l}
x-y=a, \\
x^{2}-y^{2}=b
\end{array}\right.
$$

над этим полем при $a \neq 0$ эквивалентна системе

$$
\left\{\begin{array}{l}
x-y=a, \\
x+y=\frac{b}{a},
\end{array}\right.
$$

которая имеет единственное решение.

Таким образом, рассматриваемая парабола является полуразностным, следовательно, 2-редким подмножеством в $G F(q)^{2}$.

Пример из п. (i.b) предложен в [39], [40] (доказательство в целом аналогично доказательству п. (i.a)). 
Утверждение п. (ii.a) фактически доказано У. Брауном в [58] для случая простого числа $q$. Более точно, он доказал, что пересечение любых трех различных сфер $S(a, b, c)=\left\{(x, y, z) \mid(x-a)^{2}+(y-b)^{2}+(z-c)^{2}=\gamma\right\}$ состоит не более чем из двух точек. Это рассуждение проходит и в общем случае.

Теперь, если предположить, что сфера $S(0,0,0)$ не является 3-редким подмножеством, т.е. $A+B \subset S(0,0,0)$, где $|A|=|B|=3$, то в силу того, что произвольную сферу $S(a, b, c)$ можно представить в виде $\{(a, b, c)\}+S(0,0,0)$, получим, что множество $B$ мощности 3 содержится в каждой из сфер $S(a, b, c)$, где $(-a,-b,-c) \in A$, что противоречит доказанному Брауном.

То, что мощность $S(0,0,0)$ равна $q^{2}-q$, следует, например, из утверждения задачи 14 из [59; гл. “Алгебраическое дополнение", § 3].

Пример из п. (ii.b) предложен в [43]. Доказательство довольно громоздкое, поэтому здесь не приводится.

Утверждение п. (iii) непосредственно вытекает из результата работы [50], в которой показано, что любые $t$ различных множеств

$$
N(a)=\left\{x \mid(x-a)^{\left(q^{t}-1\right) /(q-1)}=1\right\}
$$

пересекаются не более чем по $t$ ! точкам; наоборот, любые $t !+1$ таких множеств имеют не более $t-1$ общих точек. Теперь $(t, t !+1)$-редкость множества $N(0)$ устанавливается тем же рассуждением, что и в пункте (ii). Теорема доказана.

Теорема 3 предоставляет примеры редких множеств большой мощности в многомерных векторных пространствах. Укажем способ, позволяющий такие множества трансформировать в числовые (“одномерные") редкие множества. Нам понадобится

Лемма 13. Обозначим $N_{q, t}=\left\{\sum_{i=0}^{t-1} a_{i}(2 q-1)^{i} \mid a_{i} \in E_{q} t\right\}$. Пусть $A, B \subset N$ u $A \times B \subset N_{q, t}$. Тогда существуют взаимно однозначные отображсения $\xi_{A}$ : $A \rightarrow N_{q, t} u \xi_{B}: B \rightarrow N_{q, t}$ такие, что для любых $a \in A, b \in B$ выполняется $a+b=\xi_{A}(a)+\xi_{B}(b)$.

Содержательно лемма означает, что если в записи элементов суммы Минковского $A \times B$ двух числовых множеств $A, B$ в системе счисления с основанием $2 q-1$ присутствуют только "цифры" от 0 до $q-1$, то элементы самих множеств можно видоизменить так, что их запись в указанной системе счисления также будет содержать только цифры от 0 до $q-1$, а попарные суммы при этом не изменятся.

ДоказАТЕльство. Доказательство проведем индукцией по $t$. Если $t=1$, то неизбежно $A, B \subset E_{q}$, поэтому в качестве $\xi_{A}$ и $\xi_{B}$ можно взять тождественные отображения. Доказательству индуктивного перехода предпошлем вспомогательное рассуждение.

В кольце вычетов $\mathbb{Z}_{m}=\{\overline{0}, \ldots, \overline{m-1}\}$ введем понятие отрезка $[\bar{a}, \bar{b}]$, определяемое при $a \leqslant b$ как $\{\bar{a}, \overline{a+1}, \ldots, \bar{b}\}$, а при $a>b$ как $\{\bar{a}, \ldots, \overline{m-1}, \overline{0}, \ldots, \bar{b}\}$. Длину отрезка определим как $|[\bar{a}, \bar{b}]|-1$, т.е. в первом случае как $b-a$, а во втором - как $b+m-a$. Для любого подмножества $M \subset \mathbb{Z}_{m}$ найдется отрезок минимальной длины (возможно, не один), содержащий $M$. Ясно, что оба конца такого отрезка должны принадлежать $M$. Длину минимального отрезка, 
содержащего $M$, назовем диаметром $M$ и обозначим $d(M)$. Заметим, что если $d(M) \leqslant(m-1) / 2$, то минимальный отрезок для $M$ определен однозначно и он содержится в любом содержащем $M$ отрезке длины не более $(m-1) / 2$. Легко показать, что

$$
\max \left\{d\left(M_{1}\right), d\left(M_{2}\right)\right\} \leqslant d\left(M_{1} \times M_{2}\right) \leqslant \min \left\{d\left(M_{1}\right)+d\left(M_{2}\right), m-1\right\} .
$$

Докажем следующее утверждение. Если $d\left(M_{1} \times M_{2}\right) \leqslant(m-1) / 2$, то $d\left(M_{1} \times\right.$ $\left.M_{2}\right)=d\left(M_{1}\right)+d\left(M_{2}\right)$. При этом если $[\bar{a}, \bar{b}]$ и $[\bar{c}, \bar{d}]-$ отрезки минимальной длины для множеств $M_{1}$ и $M_{2}$ соответственно, то для множества $M_{1} \times M_{2}$ минимальным является отрезок

$$
\rho=[\overline{(a+c) \bmod m}, \overline{(b+d) \bmod m}]
$$

(напомним, при рассматриваемых ограничениях все минимальные отрезки определены однозначно).

Случай, когда одно из множеств имеет нулевой диаметр, является тривиальным, поэтому без ограничения общности будем считать, что $0<d\left(M_{1}\right) \leqslant d\left(M_{2}\right)$. В силу того, что $d\left(M_{2}\right) \leqslant(m-1) / 2$, отрезок $\rho$ имеет длину $d\left(M_{1}\right)+d\left(M_{2}\right)$ и содержит множество $M_{1} \times M_{2}$. Предположим, что этот отрезок не является минимальным для $M_{1} \times M_{2}$. Поскольку все вычеты

$$
\overline{(a+c) \bmod m}, \quad \overline{(b+c) \bmod m}, \quad \overline{(a+d) \bmod m}, \quad \overline{(b+d) \bmod m}
$$

принадлежат множеству $M_{1} \times M_{2}$, то любой отрезок, содержащий данное множество, содержит при $d\left(M_{2}\right)>d\left(M_{1}\right)$ один из отрезков

$$
\begin{array}{r}
\rho=[\overline{(a+c) \bmod m}, \overline{(b+d) \bmod m}], \quad[\overline{(b+c) \bmod m}, \overline{(a+c) \bmod m}], \\
{[\overline{(a+d) \bmod m}, \overline{(b+c) \bmod m}], \quad[\overline{(b+d) \bmod m}, \overline{(a+d) \bmod m}],}
\end{array}
$$

а при $d\left(M_{1}\right)=d\left(M_{2}\right)$ - один из перечисленных отрезков, не считая третьего.

Второй и четвертый отрезок имеют длину $m-d\left(M_{1}\right)>(m-1) / 2$, а третий отрезок при $d\left(M_{2}\right)>d\left(M_{1}\right)$ имеет длину $m-\left(d\left(M_{2}\right)-d\left(M_{1}\right)\right)>(m-1) / 2$. Следовательно, минимальный отрезок для $M_{1} \times M_{2}$ должен содержать отрезок $\rho$, что противоречит предположению.

Перейдем к доказательству индуктивного перехода леммы. Пусть $t \geqslant 2$. Предположим, что утверждение леммы справедливо при подстановке $t-1$ вместо $t$. Определим множество "младших разрядов" $A_{0}=\{\overline{a \bmod (2 q-1)} \mid a \in A\}$ и аналогично - множество $B_{0}$. По условию леммы в кольце вычетов $\mathbb{Z}_{2 q-1}$ справедливо вложение $A_{0} \times B_{0} \subset[\overline{0}, \overline{q-1}]$. Пусть $\rho_{A}=\left[\overline{l_{A}}, \overline{r_{A}}\right]$ и $\rho_{B}=\left[\overline{l_{B}}, \overline{r_{B}}\right]-$ минимальные отрезки для $A_{0}$ и $B_{0}$ соответственно. Тогда по доказанному выше отрезок

$$
\rho=\left[\overline{\left(l_{A}+l_{B}\right) \bmod (2 q-1)}, \overline{\left(r_{A}+r_{B}\right) \bmod (2 q-1)}\right]
$$

является минимальным для $A_{0} \times B_{0}$. Ясно, что $\rho \subset[\overline{0}, \overline{q-1}]$ (поскольку любой содержащий некоторое подмножество $\mathbb{Z}_{m}$ отрезок длины не более $(m-1) / 2$ содержит также минимальный отрезок для этого подмножества). 
Теперь заметим, что если $l_{A}=0$ или $l_{B}=0$, то $r_{A}, r_{B} \leqslant q-1$. Действительно, пусть, например, $l_{A}=0$. Тогда $l_{B} \leqslant q-1$ и $r_{A} \leqslant q-1$. Если предположить, что $r_{B} \geqslant q$, то в случае $l_{B} \leqslant r_{A}$ получаем

$$
d\left(A_{0} \times B_{0}\right)=\left(r_{A}-l_{A}\right)+\left(r_{B}-l_{B}\right) \geqslant r_{B} \geqslant q
$$

(противоречие с условием $\left.d\left(A_{0} \times B_{0}\right) \leqslant q-1\right)$, а в случае $l_{B} \geqslant r_{A}$ получаем

$$
q \leqslant r_{A}+r_{B}=\left(l_{A}+l_{B}\right)+d\left(A_{0} \times B_{0}\right) \leqslant l_{B}+q-1 \leqslant 2 q-2
$$

(противоречие с условием $\rho \subset[\overline{0}, \overline{q-1}])$. Поэтому $r_{B} \leqslant q-1$.

Далее заметим, что если $\overline{0} \in\left(\rho_{A} \cap \rho_{B}\right)$, то $\overline{0} \in \rho$, следовательно, $l_{A}=l_{B}=0$. Поэтому если $l_{A} \neq 0$ и $l_{B} \neq 0$, то $\overline{0} \notin\left(\rho_{A} \cap \rho_{B}\right)$, иными словами, либо $0<l_{A} \leqslant r_{A}$, либо $0<l_{B} \leqslant r_{B}$.

Следовательно, в любом случае выполнено либо $l_{A} \leqslant r_{A}$, либо $l_{B} \leqslant r_{B}$. Без ограничения общности предположим, что выполнено первое неравенство - оно означает, что для любого $a \in A$ верно $a \geqslant l_{A}$. Определим отображения $\xi_{A}^{\prime}$ и $\xi_{B}^{\prime}$ следующим образом: $\xi_{A}^{\prime}(a)=a-l_{A}, \xi_{B}^{\prime}(b)=b+l_{A}$.

Ясно, что $\xi_{A}^{\prime}(a)+\xi_{B}^{\prime}(b)=a+b$. В силу $l_{\xi_{A}^{\prime}(A)}=0$ имеем также $0 \leqslant$ $l_{\xi_{B}^{\prime}(B)}, r_{\xi_{A}^{\prime}(A)}, r_{\xi_{B}^{\prime}(B)} \leqslant q-1$ (где $l_{\xi_{A}^{\prime}(A)}$ и $r_{\xi_{A}^{\prime}(A)}$ определяются по аналогии с $l_{A}$ и $\left.r_{A}\right)$. Как следствие, имеет место $\xi_{A}^{\prime}(A), \xi_{B}^{\prime}(B) \subset(2 q-1) N \times E_{q}$, что значит: элемент а любого из множеств $\xi_{A}^{\prime}(A), \xi_{B}^{\prime}(B)$ можно записать в виде $a=(2 q-1) a^{\prime}+a^{\prime \prime}$, где $a^{\prime \prime} \in E_{q}, a^{\prime} \in N$. Определим новые множества

$$
\begin{aligned}
& A^{\prime}=\left\{a^{\prime} \mid a=(2 q-1) a^{\prime}+a^{\prime \prime} \in \xi_{A}^{\prime}(A), a^{\prime \prime} \in E_{q}\right\}, \\
& B^{\prime}=\left\{b^{\prime} \mid b=(2 q-1) b^{\prime}+b^{\prime \prime} \in \xi_{B}^{\prime}(B), b^{\prime \prime} \in E_{q}\right\} .
\end{aligned}
$$

Для любых $a^{\prime} \in A^{\prime}$ и $b^{\prime} \in B^{\prime}$ выполнено $a^{\prime}+b^{\prime}=\lfloor(a+b) /(2 q-1)\rfloor$ при некоторых $a \in \xi_{A}^{\prime}(A)$ и $b \in \xi_{B}^{\prime}(B)$, потому что $0 \leqslant a^{\prime \prime}+b^{\prime \prime} \leqslant 2 q-2$, где $a^{\prime \prime}=a \bmod (2 q-1)$ и $b^{\prime \prime}=b \bmod (2 q-1)$, и переносов из младшего разряда (в системе счисления с основанием $2 q-1$ ) не возникает. Поэтому $A^{\prime} \times B^{\prime} \subset$ $N_{q, t-1}$. Следовательно, по индуктивному предположению существуют взаимно однозначные отображения $\xi_{A}^{\prime \prime}: A^{\prime} \rightarrow N_{q, t-1}$ и $\xi_{B}^{\prime \prime}: B^{\prime} \rightarrow N_{q, t-1}$, сохраняющие суммы.

Окончательно положим $\xi_{A}(a)=(2 q-1) \xi_{A}^{\prime \prime}\left(a^{\prime}\right)+a^{\prime \prime}$, где $\xi_{A}^{\prime}(a)=(2 q-1) a^{\prime}+a^{\prime \prime}$, $a^{\prime \prime} \in E_{q}$, и аналогично определим $\xi_{B}(b)$. По построению отображение $\xi_{A}$ (аналогично $\xi_{B}$ ) состоит в прибавлении (вычитании) некоторой целой константы, поэтому, в частности, является взаимно однозначным. Лемма доказана.

ЗАмечАниЕ 3. Утверждение леммы останется справедливым, если сумму по степеням $2 q-1$ в определении множества $N_{q, t}$ заменить суммой по степеням произвольного числа $Q \geqslant 2 q-1$.

Рассмотрим отображение $\psi_{q, s, t}$ из $E_{q}^{s t}$ в $E_{(2 q-1)^{t}}^{s}$, переводящее вектор $\left(a_{0}\right.$, $\left.\ldots, a_{s t-1}\right)$ в вектор

$$
\left(\sum_{i=0}^{t-1} a_{i}(2 q-1)^{i}, \sum_{i=0}^{t-1} a_{t+i}(2 q-1)^{i}, \ldots, \sum_{i=0}^{t-1} a_{(s-1) t+i}(2 q-1)^{i}\right) .
$$


Теорема 4. Если подмножество $M \subset E_{q}^{s t}$ полугруппы $\left(N^{s t},+\right)$ является $(k, l)$-редким, то подмножество $\psi_{q, s, t}(M)$ полугруппь $\left(N^{s},+\right)$ также является $(k, l)$-редким.

ДокАЗАТЕльство. Через $\operatorname{pr}_{i}(A)$ будем обозначать множество $i$-х компонент множества $A \subset N^{s}$, а именно $\operatorname{pr}_{i}(A)=\left\{a_{i} \mid\left(a_{0}, \ldots, a_{s-1}\right) \in A\right\}$. Оператор $\operatorname{pr}_{i}$ обладает очевидным свойством: $\operatorname{pr}_{i}(A \times B)=\operatorname{pr}_{i}(A) \times \operatorname{pr}_{i}(B)$.

Проверим, что отображение $\psi_{q, s, t}$ сохраняет редкость множества. Действительно, согласно лемме 13 если для некоторых множеств $A, B \subset N^{s}$ верно, что множество $A \times B$ содержится в $\psi_{q, s, t}(M)$, то для каждого $i=1, \ldots, s$ найдется пара взаимно однозначных отображений $\xi_{i, A}: \operatorname{pr}_{i}(A) \rightarrow N_{q, t}$ и $\xi_{i, B}: \operatorname{pr}_{i}(B) \rightarrow$ $N_{q, t}$, обладающих свойством $\xi_{i, A}(a)+\xi_{i, B}(b)=a+b$ (где $N_{q, t}$ - из леммы 13$)$. Следовательно, вектор-отображения $\xi_{A}=\left(\xi_{0, A}, \ldots, \xi_{s-1, A}\right)$ и $\xi_{B}=\left(\xi_{0, B}, \ldots\right.$, $\left.\xi_{s-1, B}\right)$ взаимно однозначно отображают соответственно множество $A$ и множество $B$ в $N_{q, t}^{s}=\psi_{q, s, t}\left(E_{q}^{s t}\right)$ и также обладают свойством $\xi_{A}(a)+\xi_{B}(b)=a+b$.

Теперь, поскольку отображение $\psi_{q, s, t}^{-1}$ сохраняет суммы и является взаимно однозначным, получаем, что $\psi_{q, s, t}^{-1}\left(\xi_{A}(A)\right) \times \psi_{q, s, t}^{-1}\left(\xi_{B}(B)\right) \subset M$ и при этом

$$
\left|\psi_{q, s, t}^{-1}\left(\xi_{A}(A)\right)\right|=\left|\xi_{A}(A)\right|=|A|, \quad\left|\psi_{q, t}^{-1}\left(\xi_{B}(B)\right)\right|=\left|\xi_{B}(B)\right|=|B| .
$$

Теорема доказана.

Еще один способ конструирования редких множеств будет указан в лемме 14.

\section{§ 6. Построение многочленов высокой монотонной сложности}

Выбирая в качестве mon $f_{1}$ множество Сидона в $E_{m}$ мощности асимптотически $\sqrt{m}$ и применяя теорему 1 , пп. (i), (iii), получаем пример монотонного многочлена $f_{1}$ одной переменной, $\operatorname{deg} f_{1}<m$, такого, что $L_{+}\left(f_{1}\right) \gtrsim \sqrt{m}$ и $L_{\times}\left(f_{1}\right) \gtrsim 2 \sqrt[3]{m}$

При $m=d^{n}$ путем кронекеровой замены переменных $x_{i}=x^{d^{i}}$ по указанному многочлену строится многочлен $\widehat{f}_{1}$ от $n$ переменных степени не выше $d-1$ по каждой переменной, для которого при $d^{n} \rightarrow \infty$ автоматически выполняются оценки сложности $L_{+}\left(\widehat{f}_{1}\right) \gtrsim d^{n / 2}, L_{\times}\left(\widehat{f}_{1}\right) \gtrsim 2 d^{n / 3}$.

Аналогичные оценки сложности можно получить, используя множества из п. (i) теоремы 3. Более высокие оценки позволяет получить п. (ii).

Пункт (ii.a) теоремы 3 позволяет указать 3 -редкое множество mon $f_{2}$ мощности асимптотически $m^{2}$ в $E_{m}^{3} \subset N^{3}$. Тогда для соответствующего многочлена $f_{2}$ от трех переменных степени не выше $m-1$ по каждой переменной из пп. (i) и (iii) теоремы 1 вытекают оценки сложности $L_{+}\left(f_{2}\right) \gtrsim(1 / 8) m^{2}$, $L_{\times}\left(f_{2}\right) \gtrsim 2^{-4 / 5} m^{6 / 5}$. Как следствие, можно указать многочлен $f_{2}$ от $3 n$ переменных степени не выше $d-1$ по каждой переменной, для которого при $d^{n} \rightarrow \infty$ справедливы оценки $L_{+}\left(\widehat{f}_{2}\right) \gtrsim(1 / 8) d^{2 n}, L_{\times}\left(\widehat{f_{2}}\right) \gtrsim 2^{-4 / 5} d^{6 n / 5}$.

Эти результаты, с точностью до мультипликативных постоянных в некоторых оценках и способа вывода, получены в работе [43]. Пункт (iii) теоремы 3 и теорема 4 позволяют установить более сильный результат. 
Теорема 5. Пусть $m \geqslant 2$ и $n \geqslant 1$. Тогда можно эффективно указать монотонный многочлен $f$ от $n$ переменных степени не выше $m-1$ по каждой из переменных такой, что при $m^{n} \rightarrow \infty$

$$
L_{+}(f)=\Omega\left(m^{n(1-o(1))}\right), \quad L_{\times}(f)=\Omega\left(m^{n(1 / 2-o(1))}\right) .
$$

ДокАЗАтельство. Пусть $n=1$. Выберем параметры $q$ и $t$ из условий: $q-$ простое число, $(2 q-1)^{t} \leqslant m$ и $q^{t-1} /(t !)^{2}=m^{1-o(1)}$, и определим множество mon $f$ как образ соответствующего редкого множества из п. (iii) теоремы 3 под действием отображения $\psi_{q, 1, t}$, см. (2). Согласно теореме 4 построенное множество является $(t, t !+1)$-редким. Оценки сложности многочлена $f$ следуют из пп. (i), (iii) теоремы 1.

Пример многочлена $n>1$ переменных $x_{0}, \ldots, x_{n-1}$ получается из построенного многочлена одной переменной $x$ степени $m^{n}-1$ подстановкой Кронекера $x_{i}=x^{m^{i}}$.

Ненулевые коэффициенты в многочленах, о которых идет речь, могут быть произвольными положительными числами - нижние оценки, извлекаемые из теоремы 1 , зависят только от множества mon $f$. Ограничим рассмотрение многочленами с коэффициентами 0 и 1 и покажем их эффективность, а именно то, что булевы функции, выражающие коэффициент многочлена через степень соответствующего монома, имеют полиномиальную сложность.

Реализацию указанной булевой функции $c_{f}\left(d_{n-1}, \ldots, d_{0}\right)$ можно свести к следующим шагам (через $d_{i}$ обозначается двоичная запись степени переменной $x_{i}$ в мономе).

1) Вычисление двоичного числа $d$ по своему представлению $\left[d_{n-1}, \ldots, d_{0}\right]_{m}$ в $m$-ичной системе счисления (т.е. сведение к многочлену одной переменной, см. выше).

2) Представление числа $d$ в системе счисления с основанием $2 q-1$, обозначим его через $\left[a_{t-1}, \ldots, a_{0}\right]_{2 q-1}$.

3) Проверка выполнения неравенств $0 \leqslant a_{i}<q$. Если какое-то из них не выполняется, то значение функции $c_{f}$ полагается равным 0 .

4) Если неравенства п. 3) выполнены, то вектор $\left(a_{t-1}, \ldots, a_{0}\right)$ интерпретируется как элемент $X$ поля $G F(q)^{t}$. Вычисляется $X^{\left(q^{t}-1\right) /(q-1)}$ и функции $c_{f}$ присваивается значение 1 в том случае, если результат равен $1 \in G F(q)^{t}$, и значение 0 в противном случае.

Указанные шаги можно реализовать схемой сложности $b \log O(1) b$ относительно размера $b=\Theta\left(\log m^{n}\right)$ входа функции $c_{f}$, если для переходов между системами счисления использовать быстрый алгоритм Шёнхаге (см., например, [14]), а для возведения в степень специального вида в конечном поле метод аддитивных цепочек (см., например, [60]).

Теорема доказана.

Поскольку аддитивная монотонная сложность любого многочлена $n$ переменных степени не выше $m-1$ по каждой из переменных не превосходит $m^{n}$, а мультипликативная монотонная сложность по порядку не превосходит $m^{n / 2}$, то оценки теоремы близки к наилучшим возможным. 
В частных случаях $m=2$ и $n=1$ теорема указывает примеры соответственно мультилинейного многочлена и многочлена одной переменной высокой монотонной сложности.

ЗАмЕчАНИЕ 4. Для аддитивной сложности (в полном базисе) класса всех мультилинейных многочленов $n$ переменных с коэффициентами 0,1 из [22] вытекает нижняя оценка $\Omega\left(2^{n / 2} / n\right)$. Из теоремы 5 следует, что для монотонного базиса можно эффективно построить мультилинейный многочлен $n$ переменных с коэффициентами 0,1 аддитивной сложности $\Omega\left(2^{(1-o(1)) n}\right)$.

\section{§ 7. Некоторые верхние оценки}

В этом пункте мы рассмотрим вопрос о том, насколько точными являются оценки теоремы 1 . Ясно, что если $l$ не растет с ростом $n$, то оценка п. (i) для $L_{+}(f)$ является точной по порядку, поскольку для любого многочлена $f$ справедливо $L_{+}(f) \leqslant|\operatorname{mon} f|-1$, а в случае $k=l=2$ оценка (i) является просто точной.

Покажем теперь, что оценки пп. (ii) и (iii) также существенно улучшить, вообще говоря, нельзя.

ТЕОРема 6. Для любого $n$ nри $k=2$ и любого $n>1$ nри $k>2$ существует $k$-редкое множество mon $f \subset E_{m}^{n}$ maкое, что $|\operatorname{mon} f| \geqslant m^{c_{k} n^{\log _{2} 3-1}} u$

$$
L_{\times}(\operatorname{mon} f) \lesssim \begin{cases}\Theta\left(|\operatorname{mon} f|^{(k+1) /(2 k)}\right), & k>3, \\ 3|\operatorname{mon} f|^{3 / 5}, & k=3, \\ 3|\operatorname{mon} f|^{2 / 3}, & k=2 .\end{cases}
$$

ДоказАтельство. Если $A$ - конечное множество, то через $A^{*}$ будем обозначать множество одноэлементных подмножеств $A$.

Лемма 14. (i) Пусть $A_{1}=\left\{a_{1}, \ldots, a_{p}\right\} \subset E_{m}^{n_{1}} u A_{2}=\left\{b_{1}, \ldots, b_{q}\right\} \subset E_{m}^{n_{2}}-$ $k$-редкие подмножества и пусть $\left(\mu_{i, j}\right)-l$-редкая булева $(p \times q)$-матрица, $n=$ $n_{1}+n_{2}, k \leqslant l$. Тогда множество $A=\left\{\left(a_{i}, b_{j}\right) \mid \mu_{i, j}=1\right\} \subset E_{m}^{n}$ является $((k-1)(l-1)+1)-$ редким. При этом

$$
L_{\times}(A) \leqslant L_{\times}\left(A_{1}^{*}\right)+L_{\times}\left(A_{2}^{*}\right)+\left|A_{1}\right|, \quad L_{\times}\left(A^{*}\right) \leqslant L_{\times}\left(A_{1}^{*}\right)+L_{\times}\left(A_{2}^{*}\right)+|A| .
$$

(ii) Пусть $A_{1}=\left\{a_{1}, \ldots, a_{p}\right\} \subset E_{m}^{n} u A_{2}=\left\{b_{1}, \ldots, b_{q}\right\} \subset E_{m}^{n}$ - k-редкие подмножества, и пусть $\left(\mu_{i, j}\right)-l$-редкая булева $(p \times q)$-матрица. Тогда множество $A=\left\{a_{i}+(2 m-1) b_{j} \mid \mu_{i, j}=1\right\} \subset E_{2 m^{2}}^{n}$ является $((k-1)(l-1)+1)$-редким. При этом

$$
\begin{aligned}
& L_{\times}(A) \leqslant L_{\times}\left(A_{1}^{*}\right)+L_{\times}\left(A_{2}^{*}\right)+\left|A_{1}\right|+2 n \log _{2} m, \\
& L_{\times}\left(A^{*}\right) \leqslant L_{\times}\left(A_{1}^{*}\right)+L_{\times}\left(A_{2}^{*}\right)+|A|+2 n \log _{2} m .
\end{aligned}
$$

ДокАЗАтельство. Положим $r=(k-1)(l-1)+1$. Проверим, что множество $A$ из п. (i) является $r$-редким. Предположим противное. Тогда найдутся различные векторы $c_{1}, \ldots, c_{r}, c_{i}=\left(c_{i, 1}, c_{i, 2}\right), c_{i, j} \in E_{m}^{n_{j}}$, и различные векторы $d_{1}, \ldots, d_{r}, d_{i}=\left(d_{i, 1}, d_{i, 2}\right), d_{i, j} \in E_{m}^{n_{j}}$, такие, что

$$
\left\{c_{1}, \ldots, c_{r}\right\} \times\left\{d_{1}, \ldots, d_{r}\right\} \subset A .
$$


Обозначим

$$
C_{j}=\left\{c_{1, j}, \ldots, c_{r, j}\right\}, \quad D_{j}=\left\{d_{1, j}, \ldots, d_{r, j}\right\}, \quad j=1,2 .
$$

Тогда для каждого $j=1,2$ справедливо включение $C_{j} \times D_{j} \subset A_{j}$.

Так как $A_{1}-k$-редкое подмножество, то без ограничения общности можно считать, что $\left|D_{1}\right|<k$. Тогда (по принципу Дирихле) найдутся не менее $l$ одинаковых векторов $d_{i, 1}$. Без ограничения общности будем считать, что $d_{1,1}=\cdots=d_{l, 1}$. Но тогда все векторы $d_{1,2}, \ldots, d_{l, 2}$ должны быть различны (потому что все векторы $d_{i}$ различны), следовательно, $\left|D_{2}\right| \geqslant l \geqslant k$. Поэтому из $k$-редкости множества $A_{2}$ следует, что $\left|C_{2}\right|<k$, т.е., рассуждая аналогично, можно считать, что $c_{1,2}=\cdots=c_{l, 2}$ и, как следствие, все векторы $c_{1,1}, \ldots, c_{l, 1}$ различны. Из доказанного следует, что

$$
\left\{c_{1}, \ldots, c_{r}\right\} \times\left\{d_{1}, \ldots, d_{r}\right\} \supset\left\{\left(c_{i, 1}+d_{1,1}, c_{1,2}+d_{j, 2}\right) \mid i, j=1, \ldots, l\right\},
$$

откуда получаем, что матрица $\left(\mu_{i, j}\right)$ содержит единичную $(l \times l)$-подматрицу. Противоречие.

Проверим соотношения для сложности. Реализуя все векторы $\left(a_{i}, \overrightarrow{0}\right)$ и $\left(\overrightarrow{0}, b_{j}\right)$ с мультипликативной сложностью $L_{\times}\left(A_{1}^{*}\right)$ и $L_{\times}\left(A_{2}^{*}\right)$ соответственно, и используя тождество $\left\{\left(a_{i}, b_{j}\right)\right\}=\left\{\left(a_{i}, \overrightarrow{0}\right)\right\} \times\left\{\left(\overrightarrow{0}, b_{j}\right)\right\}$, получаем заявленную оценку для $L_{\times}\left(A^{*}\right)$. Для вывода первой оценки воспользуемся формулой

$$
A=\bigvee_{i=1}^{p}\left\{\left(a_{i}, \overrightarrow{0}\right)\right\} \times\left(\bigvee_{\mu_{i, j}=1}\left\{\left(\overrightarrow{0}, b_{j}\right)\right\}\right)
$$

Докажем утверждение (ii). Согласно доказанному в п. (i) множество $A^{\prime}=$ $\left\{\left(a_{i}, b_{j}\right) \mid \mu_{i, j}=1\right\} \subset E_{m}^{2 n}$ является $r$-редким. Тогда по теореме 4 множество $A=\psi_{m, n, 2}\left(A^{\prime}\right)$ также является $r$-редким.

Проверим соотношения для сложности. Реализация множества $\{2 m-1\}$ требует не более $2 \log _{2} m$ умножений, если построить бинарную аддитивную цепочку для числа $2 m-1$ (см., например, [14]). Поэтому набор векторов $\left\{(2 m-1) e_{0}\right.$, $\left.\ldots,(2 m-1) e_{n-1}\right\}$, где $e_{0}, \ldots, e_{n-1}$ - единичные (базисные) векторы в $N^{n}$, можно реализовать за $2 n \log _{2} m$ умножений. После этого все одноэлементные множества $\left\{a_{i}\right\}$ и $\left\{(2 m-1) b_{j}\right\}$ реализуются со сложностью $L_{\times}\left(A_{1}^{*}\right)+L_{\times}\left(A_{2}^{*}\right)$. Теперь требуемые соотношения вытекают из формул

$$
\begin{gathered}
\left\{a_{i}+(2 m-1) b_{j}\right\}=\left\{a_{i}\right\} \times\left\{(2 m-1) b_{j}\right\}, \\
A=\bigvee_{i=1}^{p}\left\{a_{i}\right\} \times\left(\bigvee_{\mu_{i, j}=1}\left\{(2 m-1) b_{j}\right\}\right) .
\end{gathered}
$$

Лемма доказана.

Вернемся непосредственно к доказательству теоремы 6. Доказанная лемма позволяет строить редкие множества достаточно большой мощности и вместе с тем относительно небольшой сложности. Из результата Пиппенджера [61] об аддитивной сложности набора числовых векторов для произвольного множества $A \subset E_{m}^{n}$ вытекает оценка

$$
L_{\times}(A) \leqslant L_{\times}\left(A^{*}\right) \leqslant n \log _{2} m+(1+o(1)) \frac{|A| n \log _{2} m}{\log _{2}\left(|A| n \log _{2} m\right)}+O(|A|) .
$$


Поэтому если в п. (ii) леммы 14 дополнительно потребовать выполнения условия $|A|=\Omega\left(\max \left\{\left|A_{1}\right|,\left|A_{2}\right|\right\} n \log _{2} m\right)$, то получим (реализуя $A_{1}^{*}$ и $A_{2}^{*}$ методом Пиппенджера), что $L_{\times}\left(A^{*}\right) \lesssim|A|$.

Далее мы будем опираться на следующие известные факты: множество $E_{m}$ содержит 2-редкое подмножество мощности, асимптотически равной $\sqrt{m}$; существует $k$-редкая $(n \times n)$-матрица с весом $\Omega_{k}\left(n^{(2 k) /(k+1)}\right)($ см. [62]), в случае $k=3$ - матрица с весом $(1+o(1)) n^{5 / 3}$ (см. [54]), а в случае $k=2-$ с весом $(1+o(1)) n^{3 / 2}$ (см. также [62]).

Теперь, рекурсивно применяя п. (i) леммы 14 с параметрами $k=l=2$, $n_{1}=\lfloor n / 2\rfloor$ и $n_{2}=\lceil n / 2\rceil$, для любого $n \geqslant 2$ можно построить 2-редкое подмножество $A$ множества $E_{m}^{n}$ с мощностью $\Theta\left(m^{1 / 2(3 / 2)^{\left\lfloor\log _{2} n\right\rfloor}}\right)$ и сложностью $L_{\times}\left(A^{*}\right) \lesssim|A|$ (при $\left.m \rightarrow \infty\right)$.

В случае $n=1$ воспользуемся п. (ii) леммы 14 (с подстановкой $\lfloor\sqrt{m / 2}\rfloor$ в качестве параметра $m$ ) и построим 2-редкое подмножество $A$ множества $E_{m}$ мощности $\Theta\left(m^{3 / 8}\right)$ и сложности $L_{\times}\left(A^{*}\right) \lesssim|A|$.

Наконец, воспользуемся первой (при $n>1$, полагая $n_{1}=\lfloor n / 2\rfloor$ и $n_{2}=\lceil n / 2\rceil$ ) или второй (при $n=1$ и $k=2$, с параметром $\lfloor\sqrt{m / 2}\rfloor$ ) конструкцией леммы 14 , выбирая в качестве множеств $A_{i}$ построенные выше 2-редкие множества (или их подмножества) такие, что $\left|A_{1}\right|=\left|A_{2}\right|$, а в качестве матрицы $\left(\mu_{i, j}\right)$ - упомянутые выше $k$-редкие матрицы большого веса. В обоих случаях получаем $k$-редкое множество $A$ с оценкой сложности $L_{\times}(A) \lesssim 3\left|A_{1}\right|$. При этом по построению в случае $n>1$ и $k>3$ выполнено $|A| \asymp\left|A_{1}\right|^{(2 k) /(k+1)}$, в случае $n>1$ и $k=3-|A| \sim\left|A_{1}\right|^{5 / 3}$, в случае $k=2-|A| \sim\left|A_{1}\right|^{3 / 2}$. Теорема доказана.

Как следствие, получаем, что в случае $k=2$ и $k=3$ оценки теоремы 1 являются точными по порядку (так как $k$-редкое подмножество является $(k, l)$ редким).

ЗАмечАнИЕ 5 . Константу 3 в оценке теоремы 6 при $k=3$ можно уточнить до $5 \sqrt[5]{2 / 27} \approx 2,971 \ldots$ Для этого нужно применить п. (i) леммы $14 \mathrm{k}$ множествам $A_{i}$ таким, что $4\left|A_{1}\right| \sim 3\left|A_{2}\right|$ и выбрать в качестве $\left(\mu_{i, j}\right) 3$-редкую подматрицу матрицы Брауна (она имеет вес $(1+o(1))\left|A_{1}\right|\left|A_{2}\right|^{2 / 3}$ ).

ЗАмЕчаниЕ 6. Если верна гипотеза [62] о $k$-редких матрицах, то при $k>3$ экспоненту $(k+1) /(2 k)$ в верхней оценке для $L_{\times}(\operatorname{mon} f)$ теоремы 6 можно заменить на $k /(2 k-1)$. Однако и такая оценка оказывается выше по порядку, чем соответствующая нижняя оценка из теоремы 1.

\section{§ 8. Редкие подмножества и редкие матрицы}

Очевидно, $(k, l)$-редкому множеству $M \subset \mathbb{Z}_{m}^{n}$ соответствует булева $(k, l)$ редкая и одновременно $(l, k)$-редкая симметричная относительно главной диагонали булева матрица $\left(\mu_{\alpha, \beta}\right)$ размера $m^{n} \times m^{n}$ (строки и столбцы нумеруются элементами $\left.\mathbb{Z}_{m}^{n}\right)$, которая определяется условием

$$
\mu_{\alpha, \beta}=1 \quad \Longrightarrow \quad \alpha+\beta \in M .
$$

Такая матрица оказывается в определенном смысле циклической, а при $n=1-$ циркулянтной (состоящей из единичных циклических диагоналей). 
Свойство цикличности проявляется в том, что матрицу $\left(\mu_{\alpha, \beta}\right)$ можно рассматривать как матрицу умножения на некоторый элемент кольца $K_{m, n}=$ $\mathbb{R}\left[x_{1}, \ldots, x_{n}\right] /\left(x_{1}^{m}-1, \ldots, x_{n}^{m}-1\right)$. В частности, циркулянтная матрица является матрицей циклической свертки с некоторым постоянным вектором. Покажем это.

Для краткости при любом $\alpha=\left(\alpha_{1}, \ldots, \alpha_{n}\right) \in \mathbb{Z}_{m}^{n}$ введем обозначение $X^{\alpha}=$ $x_{1}^{\alpha_{1}} \cdots x_{n}^{\alpha_{n}}$. Определим элемент $a=\sum_{\alpha \in \mathbb{Z}_{m}^{n}} a_{\alpha} X^{\alpha}$ кольца $K_{m, n}$ соотношением на коэффициенты: $a_{\alpha}=1$, если $\alpha \in M$, и $a_{\alpha}=0$ иначе. Таким образом, $\mu_{\alpha, \beta}=a_{\alpha+\beta}$ при любых $\alpha, \beta \in \mathbb{Z}_{m}^{n}$.

Умножим строку с номером $\alpha$ матрицы $\left(\mu_{\alpha, \beta}\right)$ на вектор коэффициентов произвольного элемента $b=\sum_{\beta \in \mathbb{Z}_{m}^{n}} b_{\beta} X^{\beta}$ кольца $K_{m, n}$ такой, что в нумерации его компонент, совпадающей с нумерацией столбцов матрицы $\left(\mu_{\alpha, \beta}\right)$, компонента с номером $\beta$ равна $b_{-\beta}$. Получим

$$
\sum_{\beta} \mu_{\alpha, \beta} b_{-\beta}=\sum_{\beta} a_{\alpha+\beta} b_{-\beta}=\sum_{\gamma+\delta=\alpha} a_{\gamma} b_{\delta},
$$

т.е. коэффициент произведения $a b \in K_{m, n}$ при $X^{\alpha}$.

Существование редкого подмножества большой мощности означает существование редкой матрицы большого веса. Интерес к построению таких матриц возникает в связи с проблемой Царанкевича (см. [63], [62], [58], [54], [50]). Редкие матрицы используются в методе [12], [9]. О редких матрицах в еще одной задаче из теории сложности булевых функций см. [64].

Примеры редких множеств пп. (ii.a) и (iii) теоремы 3 в указанном выше смысле соответствуют примерам редких матриц, построенным в работах [58], [50]. Но для того чтобы по редкой матрице можно было построить редкое подмножество (вышеуказанным способом), матрица должна удовлетворять дополнительным ограничениям, так что построение редкого подмножества является, вообще говоря, более трудной задачей, чем построение редкой матрицы.

Редкие подмножества дают даже больше. Методом теоремы 4 (см. также доказательство теоремы 5 ) из них можно эффективно получать $k$-редкие подмножества мощности $n^{1-o(1)}$ в циклической группе $\mathbb{Z}_{n}$ при медленно растущем $k$ и, как следствие, $k$-редкие циркулянтные матрицы веса $n^{2-o(1)}$. Более точно, из п. (iii) теоремы 3 (с выбором простого числа в качестве $q$ ) и теоремы 4 вытекает

СлЕДСТВИЕ 1. При любом $n$ можно эфбективно ${ }^{16}$ указать $(k, l)$-редкую ицикулянтную матричу порядка $n$ и веса $\alpha n^{2}$, где

$$
k=O\left(\sqrt{\frac{\log n}{\log \log n}}\right), \quad l, \alpha^{-1} \in 2^{O(\sqrt{\log n \log \log n})} .
$$

Неэффективным образом существование циркулянтных редких матриц большого веса доказал в [65] М. И. Гринчук, см. также [66]. Применяя теорему 1, из его результата можно вывести существование многочленов степени $n$ с коэффициентами 0,1 аддитивной монотонной сложности $n^{1-o(1)}$ и мультипликативной

\footnotetext{
${ }^{16}$ Матрица считается эффективной, если ее элементы выражаются булевой функцией от (двоичной записи) своих координат, реализуемой схемой полиномиальной сложности.
} 
монотонной сложности $n^{0,5-o(1)}$, причем эти оценки будут сильнее (в члене $o(1))$, чем те, которые вытекают из доказательства п. (iii) теоремы 5.

Укажем еще одно следствие из п. (i) теоремы 1. Для булевой матрицы $A=$ $\left(a_{i, j}\right)$ введем обозначение

$$
L_{+}(A)=\min \left\{L_{+}(B X) \mid B=\left(b_{i, j}\right), b_{i, j}>0 \Longrightarrow a_{i, j}=1\right\},
$$

где $B X-$ линейный оператор с матрицей $B$. Тогда, если $A_{n}-(k, l)$-редкая и $(l, k)$-редкая булева $(n \times n)$-матрица, то

$$
L_{+}\left(A_{n}\right) \geqslant \frac{\sum_{i, j} a_{i, j}}{h}-n,
$$

где $h=\max \left\{(k-1)^{3},(l-1)^{2}\right\}$. Для доказательства достаточно рассмотреть многочлен $f_{B}=\sum b_{i, j} x_{i} y_{j}$ и применить теорему 1 (множество mon $f_{B}$ является $(k, l)$-редким).

Отметим, впрочем, что неравенство

$$
L_{+}\left(A_{n}\right) \geqslant \frac{\sum_{i, j} a_{i, j}}{(k-1)(l-1)}-\frac{n}{l-1}
$$

легко вывести также из работы [67].

Эти неравенства применимы для получения эффективных высоких нижних оценок $L_{+}\left(A_{n} X\right)$, где $A_{n}$ - булева матрица. Достаточно в качестве $A_{n}$ взять матрицу из следствия 1 . Тогда $L_{+}\left(A_{n}\right)=n^{2-o(1)}$. Более того, для такой матрицы справедлива верхняя оценка $L\left(A_{n}\right)=O\left(n^{1+o(n)}\right)$ сложности в полном линейном базисе $\{x+y\} \cup\{c x: c \in \mathbb{R}\}$ (см., например, [64]). Поэтому для матрицы $A_{n}$ справедливо соотношение $L_{+}\left(A_{n}\right) / L\left(A_{n}\right) \geqslant n^{1-o(1)}$. Данный факт является некоторым аналогом одного результата из [64]. Из результата [23] (который, впрочем, может быть доказан методом Лупанова) вытекает верхняя оценка $L_{+}\left(A_{n}\right) / L\left(A_{n}\right) \leqslant O(n / \log n)$ для произвольной булевой матрицы.

В работе Хайнца и Зивекинга [16] эффективно построены примеры матриц сложности $\Theta\left(n^{2}\right)$, причем не в монотонном, а в полном базисе. Однако существенно, что элементы этих матриц являются алгебраическими числами. Доказательство нижних оценок для матриц в [16] проводится подобно данному там же доказательству нижних оценок сложности многочленов одной переменной. В [68] показано, как очень просто можно вывести нижние оценки сложности матриц из нижних оценок сложности многочленов одной переменной.

\section{§ 9. Монотонная и немонотонная сложность}

В этом параграфе мы покажем как можно уточнить результат [31] о возможном уменьшении сложности многочлена при добавлении в монотонный базис отрицательных констант.

Рассмотрим следующую конструкцию. Пусть $A_{1}, A_{2} \subset E_{m}^{n}-k$-редкие подмножества мощности $r$. Пусть $\left(\mu_{i, j}\right)$ - циркулянтная матрица порядка $r$ из следствия 1. Пусть множество $A$ строится из множеств $A_{1}, A_{2}$ по матрице $\left(\mu_{i, j}\right)$ одним из способов леммы $14\left(A \subset E_{m}^{2 n}\right.$, если применяется п. (i) леммы 14 , и $A \subset E_{2 m^{2}}^{n}$, если применяется п. (ii)). Справедлива 
Лемма 15. Пусть $f$ - многочлен с коэффициентами 0 и 1 такой, что $\operatorname{mon} f=A$. Пусть $k=r^{o(1)}$ и либо $n \log m=r^{o(1)}$, либо $\operatorname{deg} f=r^{o(1)}$. Тогда для монотонной сложности многочлена $f$ справедлива оченка $L_{+}(f)=\Omega\left(r^{2-o(1)}\right)$, а сложность $L(f)$ вычисления $f$ в полном базисе $\{x+y, x y\} \cup \mathbb{R}$ не превосходит $r^{1+o(1)}$.

ДокАЗАТЕЛЬСтво. Нижняя оценка $L_{+}(f)=\Omega\left(r^{2-o(1)}\right)$ следует при $k=r^{o(1)}$ из п. (i) теоремы 1. Докажем верхнюю оценку сложности реализации в полном базисе.

Воспользуемся для реализации многочлена подходящей из формул (3) и (4). Во-первых, покажем, что все мономы, соответствующие элементам множества $A_{1}$, т.е. в ранее введенных обозначениях набор многочленов $\operatorname{mon}^{-1}\left(A_{1}^{*}\right)$, можно вычислить схемой сложности $r^{1+o(1)}$ (и такой же результат справедлив для множества $\left.A_{2}\right)$.

Действительно, если $\operatorname{deg} f=r^{o(1)}$, то степень каждого монома из $\operatorname{mon}^{-1}\left(A_{1}^{*}\right)$ не превосходит $r^{o(1)}$, и все $r$ мономов можно реализовать независимо. Если $n \log m=r^{o(1)}$, то оценка сложности $r^{1+o(1)}$ следует из (5).

В вычислениях по формуле (4) для всех переменных нужно предварительно вычислить $(2 m-1)$-е степени (именно эти степени подаются на входы схемы, вычисляющей мономы с показателями из $A_{2}$ ). Сложность этого шага не превосходит $2 n \log _{2} m=r^{o(1)}$.

Следующим шагом является вычисление линейных комбинаций мономов, отвечающих множеству $A_{2}$, коэффициенты которых определяются строками матрицы $\left(\mu_{i, j}\right)$. Циркулянтная матрица является матрицей умножения на постоянный многочлен (см. предыдущий параграф). Поэтому линейное преобразование с такой матрицей выполняется при помощи алгоритма быстрого преобразования Фурье (см., например, [69]) со сложностью $O(r \log r)$.

Остается домножить мономы, соответствующие элементам множества $A_{1}$, на полученные на предыдущем шаге линейные комбинации и результаты сложить. Это выполняется со сложностью $2 r$. Лемма доказана.

Теорема 7. Пусть $m \geqslant 2$ и $n \geqslant 1$. Тогда можно эффективно указать монотонный многочлен $f$ от $n$ переменных степени не выше $m-1$ по каждой из переменных, для которого отношение сложности реализации в монотонном базисе $\{x+y, x y\} \cup \mathbb{R}_{+} \kappa$ сложности реализации в полном базисе $\{x+y, x y\} \cup \mathbb{R}$ при $m^{n} \rightarrow \infty$ не менъше чем $m^{(0,5-o(1)) n}$.

ДокАЗАтельство. Воспользуемся конструкцией леммы 15 . В качестве $A_{1}$, $A_{2}$ выберем $r^{o(1)}$-редкие подмножества экстремальной мощности $r=m^{(0.5-o(1)) n}$ в $E_{m^{\prime}}^{n^{\prime}}$, где $n^{\prime}=\lfloor n / 2\rfloor, m^{\prime}=m$ при $n \rightarrow \infty$ и $n^{\prime}=n, m^{\prime}=\lfloor\sqrt{m / 2}\rfloor$ иначе. При нечетном $n$ в первом случае построенное множество $A \subset E_{m}^{2 n^{\prime}}$ погрузим в $E_{m}^{n}$ при помощи естественного отображения $A \rightarrow(A, 0)$. Поскольку выполнено условие $n \log m=r^{o(1)}$, для многочлена $f$, mon $f=A$, согласно лемме 15 справедливы оценки $L_{+}(f)=\Omega\left(m^{n-o(n)}\right)$ и $L(f)=O\left(m^{n / 2+o(n)}\right)$.

Эффективность устанавливается так же, как и в теореме 5 , с тем отличием, что добавляется дополнительный шаг, который сводит проверку принадлежности некоторого элемента множеству $A$, построенного из множеств $A_{1}$ и $A_{2}$ 
приемом леммы 14, к проверке принадлежности двух легко определяемых элементов множествам $A_{1}$ и $A_{2}$. Теорема доказана.

ЗАмечание 7. Стандартным образом можно показать, что оценка немонотонной сложности в теореме 7 достигается уже на схеме, содержащей всего один немонотонный элемент (умножения на отрицательную константу). Для этого используется представление (произвольного) многочлена в виде разности двух монотонных многочленов. В таком представлении любая операция в полном арифметическом базисе сводится к нескольким монотонным операциям с монотонными многочленами. Немонотонная операция выполняется в самом конце вычислений, чтобы перейти к обычному представлению.

Лемма 15 также позволяет ответить на "открытый вопрос" 9 из обзорной статьи [70]: установить расхождение монотонной и немонотонной сложности для многочленов постоянной степени (в примере [31] степень была растущей).

Предварительно заметим, что вопрос на самом деле закрыт еще в статье Шнорра [29]. ${ }^{17}$ Подходящими примерами являются многочлены $S C_{n}$ и $S M_{n}$, используемые для доказательства нижних оценок сложности свертки и произведения матриц. Все же приведем еще два примера. Справедливо

СлеДСтвиЕ 2. Можно эффективно указать мультилинейный многочлен $f$

(i) степени $k(k-1)$ от $n(n-1)$ переменных, для сложности которого при $n \rightarrow \infty$ выполняются оченки $L_{+}(f)=\Omega\left(n^{2 k-o(1)}\right)$ u $L(f)=O\left(n^{k+o(1)}\right)$;

(ii) степени $2^{k}$ от $2^{k} n$ переменных, для сложности которого при $n \rightarrow \infty$ выполняются оценки $L_{+}(f)=\Omega\left(n^{2^{k}-o(1)}\right)$ и $L(f)=O\left(n^{2^{k-1}+o(1)}\right)$.

ДоказАтельство. Докажем п. (i). В качестве множеств $A_{1}$ и $A_{2}$ выберем 2-редкие подмножества $E_{2}^{C_{n}^{2}}$, соответствующие характеристическому многочлену $k$-клики [29]

$$
C L_{n, k}=\sum_{1 \leqslant i_{1}<\cdots<i_{k} \leqslant n} \prod_{1 \leqslant s<t \leqslant k} x_{i_{s}, i_{t}}
$$

Способом леммы 15 из этих множеств построим множество $A \subset E_{2}^{n(n-1)}$ и положим $f=f_{A}$. Для сложности многочлена $f$ оценки леммы 15 справедливы в силу $\operatorname{deg} f=O(1)$. Пункт (i) доказан.

Доказательство п. (ii) при $k=1$ использует многочлен $f_{B}$ из предыдущего параграфа, соответствующий подходящей редкой матрице из следствия 1. Он является примером билинейной формы $2 n$ переменных монотонной сложности $n^{2-o(1)}$ и сложности $n^{1+o(1)}$ в полном базисе. Заметим, что отношение монотонной и немонотонной сложности многочлена $f_{B}$ близко к максимально возможному для многочленов степени 2 , поскольку очевидно, что оно не может превосходить $O(n)$.

Положим $f_{B, 1}=f_{B}$. При произвольном $k$ требуемый многочлен $f=f_{B, k}$ строится способом леммы 15 из множеств $A_{1}=A_{2}=\operatorname{mon} f_{B, k-1}$ как в п. (i).

Эффективность построенных многочленов следует из эффективности многочленов $C L_{n, k}$ и $f_{B}$. Следствие доказано.

${ }^{17}$ Отметим, что фундаментальная статья [29] в обзоре [70] даже не упоминается. 
В работе [29] были получены точные значения аддитивной монотонной сложности для систем билинейных форм, соответствующих задачам умножения многочленов (т.е. свертке) и умножения матриц. Можно сформулировать обобщающее утверждение в терминах редких множеств.

Лемма 16. Пусть $\left\{g_{i}\left(x_{0}, y_{0}, \ldots, x_{n-1}, y_{n-1}\right) \mid i=1, \ldots, k\right\}$ - система билинейных форм таких, что mon $g_{i}-(1,2)$-редкое множество, mon $g_{i} \cap \operatorname{mon} g_{j}=\varnothing$ для любъх $i \neq j$. Тогда

$$
L_{+}\left(g_{1}, \ldots, g_{k}\right)=\sum_{i=1}^{k}\left|\operatorname{mon} g_{i}\right|-k, \quad L_{\times}\left(g_{1}, \ldots, g_{k}\right)=\sum_{i=1}^{k}\left|\operatorname{mon} g_{i}\right| .
$$

ДокАзАтельство. Верхние оценки очевидны. Для доказательства нижней оценки мультипликативной сложности достаточно заметить, что каждую из форм $g_{i}$ невозможно вычислить, не вычислив по отдельности все мономы, используя $\mid$ mon $g_{i} \mid$ элементов умножения (это следует из $(1,2)$-редкости), и далее учесть, что мономы у разных форм различны. Отсюда же следует и оценка аддитивной сложности, которую иначе можно получить методом из доказательства теоремы 1. Лемма доказана.

Как следствие, свертка $c_{k}\left(x_{0}, y_{0}, \ldots, x_{n-1}, y_{n-1}\right)=\sum_{i+j=k} x_{i} y_{j}$, где $k=0, \ldots$, $2 n-2$, имеет аддитивную монотонную сложность $n^{2}-2 n-1$ и мультипликативную монотонную сложность $n^{2}$. Для сравнения, в полном арифметическом базисе справедливы оценки $O(n \log n)$ и $\Theta(n)$ соответственно.

Произведение $(n \times n)$-матриц согласно лемме 16 имеет аддитивную монотонную сложность $n^{3}-n^{2}$ и мультипликативную монотонную сложность $n^{3}$. В полном базисе обе величины оцениваются сверху как $O\left(n^{\omega}\right)$, где $\omega<2.4$.

Рассматриваются и булевы аналоги этих задач, в которых вместо операций сложения и умножения используются операции дизъюнкции и конъюнкции соответственно. Если для булевого аналога задачи о монотонном вычислении матричного произведения доказаны такие же точные оценки, см. [71], то в случае монотонного вычисления булевой свертки можно указать нижнюю оценку числа дизъюнкций $\Omega\left(n^{2} / \log ^{O(1)} n\right)$ [66], а для числа конъюнкций Н. Блюмом [72] заявлена оценка $\Omega\left(n^{3 / 2}\right)$. Булев аналог леммы 16 из [71; теорема 7.1] позволяет получать нетривиальные нижние, однако, в общем случае, не точные оценки.

\section{Список литературы}

[1] М. Гэри, Д. Джонсон, Вычислительные машины и труднорешаемые задачи, Мир, М., 1982; пер. с англ.: М.R. Garey, D. S. Johnson, Computers and intractability, Freeman, San Francisco, CA, 1979.

[2] Р. Г. Нигматуллин, Сложность булевых функиий, Наука, М., 1991.

[3] О.Б. Лупанов, Асимптотические оченки сложности управляющих систем, Изд-во МГУ, М., 1984.

[4] О.Б. Лупанов, "О методах получения оценок сложности и вычисления индивидуальных функций”, Дискрет. анализ, 25 (1974), 3-18.

[5] В. М. Храпченко, "Нижние оценки сложности схем из функциональных элементов (обзор)", Кибернетический сборник. Новая серия, т. 21, Мир, М., 1984, 3-54. 
[6] Р.Г. Нигматуллин, Нижние оценки сложности и сложность универсалъных схем, Изд-во Казанского ун-та, Казань, 1990.

[7] А.А. Разборов, "Нижние оценки монотонной сложности некоторых булевых функций”, Докл. АН ССCP, 281:4 (1985), 798-801; англ. пер.: А. A. Razborov, "Lower bounds for the monotone complexity of some Boolean functions", Soviet Math. Dokl., 31:2 (1985), 354-357.

[8] А.А. Разборов, "Нижние оценки монотонной сложности логического перманента", Матем. заметки, 37:6 (1985), 887-900; англ. пер.: А. A. Razborov, "Lower bounds on monotone complexity of the logical permanent", Math. Notes, 37:6 (1985), 485-493.

[9] А. Е. Андреев, "Об одном методе получения эффективных нижних оценок монотонной сложности", Алгебра и логика, 26:1 (1987), 3-26; англ. пер.: А. E. Andreev, "A method for obtaining efficient lower bounds for monotone complexity", Algebra and Logic, 26:1 (1987), 1-18.

[10] N. Alon, R. B. Boppana, "The monotone circuit complexity of boolean functions", Combinatorica, 7:1 (1987), 1-22.

[11] А.Е. Андреев, Об одном методе получения нижних оценок сложности индивидуальных монотонных функиий, Препринт № 248 ИПМех АН СССР и МГУ, М., 1985.

[12] А. Е. Андреев, "Об одном методе получения нижних оценок сложности индивидуальных монотонных функций”, Докл. АН СCСР, 282:5 (1985), 1033-1037; англ. пер.: A. E. Andreev, "On a method for obtaining lower bounds for the complexity of individual monotone functions", Soviet Math. Dokl., 31:3 (1985), 530-534.

[13] D. Harnik, R. Raz, "Higher lower bounds on monotone size", Proceedings of the 32 Annual ACM Symposium on Theory of Computing, ACM, New York, 2000, 378-387.

[14] Д. Кнут, Искусство программирования, т. 2: Получисленные алгоритмы, Вильямс, М., 2000; пер. с англ.: D. E. Knuth, The art of computer programming, v. 2: Seminumerical algorithms, Bonn, Reading, MA, 1998.

[15] И. Фон цур Гатен, Ф. Штрассен, "Некоторые многочлены, имеющие высокую сложность вычисления", Кибернетический сборник. Новая серия, т. 20, Мир, М., 1983, 59-63; пер. с англ.: J. von zur Gathen, V. Strassen, "Some polynomials that are hard to compute", Theoret. Comput. Sci., 11:3 (1980), 331-335.

[16] И. Хайнц, М. Зивекинг, "Нижние оценки для многочленов с алгебраическими коэффициентами", Кибернетический сборник. Новая серия, т. 20, Мир, М., 1983, 46-58; пер. с англ.: J. Heintz, M. Sieveking, "Lower bounds for polynomials with algebraic coefficients", Theoret. Comput. Sci., 11:3 (1980), 321-330.

[17] Х. Штосс, "Нижние оценки сложности полиномов", Кибернетический сборник. Новая серия, т. 28, Мир, М., 1991, 168-178; пер. с англ.: Н.-J. Stoss, "Lower bounds for the complexity of polynomials", Theoret. Comput. Sci., 64:1 (1989), 15-23.

[18] W. Baur, K. Halupczok, "On lower bounds for the complexity of polynomials and their multiples", Comput. Complexity, 8:4 (1999), 309-315.

[19] M.S. Paterson, L. J. Stockmeyer, "On the number of nonscalar multiplications necessary to evaluate polynomials", SIAM J. Comput., 2 (1973), 60-66.

[20] Д.Ю. Григорьев, "Нижние оценки в алгебраической сложности вычислений", Теория сложности вычислений. I, Зап. научн. сем. ЛОМИ, 118, Изд-во "Наука", Ленинград. отд., Л., 1982, 25-82.

[21] P. Bürgisser, M. Clausen, M. A. Shokrollahi, Algebraic complexity theory, Grundlehren Math. Wiss., 315, Springer-Verlag, Berlin-Heidelberg, 1997.

[22] К. Шнорр, Ш. Ван де Виль, "Об аддитивной сложности многочленов", Кибернетический сборник. Новая серия, т. 22, Мир, М., 1985, 19-42; пер. с англ.: C.P. Schnorr, J.P. van de Wiele, "On the additive complexity of polynomials", Theoret. Comput. Sci., 10:1 (1980), 1-18. 
[23] J. E. Savage, "An algorithm for the computation of linear forms", SIAM J. Comput., 3 (1974), 150-158.

[24] V. Strassen, "Berechnungen in partiellen Algebren endlichen Typs", Computing (Arch. Elektron. Rechnen), 11:3 (1973), 181-196.

[25] С. Б. Гашков, "О сложности вычисления некоторых классов многочленов нескольких переменных", Вестн. Моск. ун-та. Сер. 1. Матем., мех., 1988, № 1, 89-91; англ. пер.: S. B. Gashkov, "On the complexity of the computation of certain classes of polynomials of several variables", Moscow Univ. Math. Bull., 43:2 (1988), 65-67.

[26] С. Б. Гашков, "О параллельном вычислении некоторых классов многочленов с растущим числом переменных", Вестн. Моск. ун-та. Сер. 1. Матем., мех., 1990, № 2, 88-92; англ. пер.: S. B. Gashkov, "On parallel evaluation of certain classes of polynomials with an increasing number of variables", Moscow Univ. Math. Bull., 45:2 (1990), 64-67.

[27] С.Б. Гашков, "Сложность реализации булевых функций схемами из функциональных элементов и формулами в базисах, элементы которых реализуют непрерывные функции”, Проблемы кибернетики, т. 37, Наука, М., 1980, 57-118.

[28] И. И. Жегалкин, "Арифметизация символической логики”, Матем. сб., 35:3-4 (1928), 311-377.

[29] К.П. Шнорр, "Нижняя оценка числа сложений в монотонных вычислениях", Кибернетический сборник. Новая серия, т. 18, Мир, М., 1981, 5-20; пер. с англ.: С.P. Schnorr, "A lower bound on the number of additions in monotone computations", Theoret. Comput. Sci., 2:3 (1976), 305-315.

[30] R. Jerrum , M. Snir, "Some exact complexity results for straight-line computations over semirings", J. Assoc. Comput. Mach., 29:3 (1982), 874-897.

[31] L. G. Valiant, "Negation can be exponentially powerful", Theoret. Comput. Sci., 12:3 (1980), 303-314.

[32] Л. Дж. Вэлиант, "Сложность вычисления перманента", Кибернетический сборник. Новая серия, т. 18, Мир, М., 1981, 125-140; пер. с англ.: L. G. Valiant, "The complexity of computing the permanent", Theoret. Comput. Sci., 8:2 (1979), 189-201.

[33] L. G. Valiant, "Completeness classes in algebra", Conference Record of the 11-th Annual ACM Symposium on Theory of Computing (Atlanta, GA, 1979), ACM, New York, 1979, 249-261.

[34] G. Malod, "The complexity of polynomials and their coefficient functions", Proc. IEEE Conf. Comput. Complexity, 13 (2007), 193-204.

[35] L. Blum, F. Cucker, M. Shub, S. Smale, Complexity and real computation, Springer-Verlag, New York, 1998.

[36] R. Sengupta, H. Venkaterswaran, "A lower bound for monotone arithmetic circuits computing 0-1 permanent", Theoret. Comput. Sci., 209:1-2 (1998), 389-398.

[37] M. Agraval, "Determinant versus permanent", Proc. International Congress of Mathematicians, т. 3, Eur. Math. Soc., Zürich, 2006, 985-997.

[38] R. Raz, "Multi-linear formulas for permanent and determinant are of super-polynomial size", Proc. of the 36th annual ACM symposium on theory of computing (Chicago, IL, USA, 2004), ACM Press, New York, 2004, 633-641.

[39] О.М. Касим-Заде, "Об арифметической сложности монотонных многочленов", Тезисы Всесоюзной конференции "Теоретические проблемы кибернетики", Изд-во Саратовского ун-та, Саратов, 1986, 68-69.

[40] О. М. Касим-Заде, "О сложности монотонных многочленов", Материаль Всесоюзного семинара по дискретной математике и ее приложениям, Изд-во МГУ, M., 1986, 136-138. 
[41] С. Е. Кузнецов, "Монотонные вычисления полиномов и схемы без нулевых цепей", Тезисы докладов VII Всесоюзной конферениии по проблемам теоретической кибернетики. Ч. 1, Иркутск, 1985, 108-109.

[42] С. Е. Кузнецов, "Схемы из функциональных элементов без нулевых цепей в базисе $\{\&, \vee,-\} "$, Изв. вузов. Матем., 1981, № 5, 56-63; англ. пер.: S. E. Kuznetsov, "Circuits of functional elements without zero chains in the basis $\{\&, v\} "$, Soviet Math. (Iz. VUZ), 25:5 (1981), 62-73.

[43] С. Б. Гашков, "Об одном методе получения нижних оценок сложности монотонных вычислений многочленов", Вестн. Моск. ун-та. Сер. 1. Матем., мех., 1987, № 5, 7-13; англ. пер.: S. В. Gashkov, "The complexity of monotone computations of polynomials", Soviet Math. (Iz. VUZ), 42:5 (1987), 1-8.

[44] R. Raz, A. Yehudayoff, "Multilinear formulas, maximal-partition discrepancy and mixed-sources extractors", J. Comput. System Sci., 77:1 (2011), 167-190.

[45] N. Nisan, A. Wigderson, "Lower bounds on arithmetic circuits via partial derivatives", Comput. Complexity, 6:3 (1996), 217-234.

[46] С.Б. Гашков, "Сложность реализации булевых функций схемами из функциональных элементов и формулами в базисах, элементы которых реализуют непрерывные функции", Докл. АН СCCP, 250:4 (1980), 782-787; англ. пер.: S. B. Gashkov, "The complexity of the realization of Boolean functions by schemes and formulas in bases consisting of continuous functions", Soviet Math. Dokl., 21:5 (1980), 186-190.

[47] G. Turán, F. Vatan, "On the computation of boolean functions by analog circuits of bounded fan-in", J. Comput. System Sci., 54:1 (1997), 199-212.

[48] A. Haken, S. A. Cook, "An exponential lower bound for the size of monotone real circuits", J. Comput. System Sci., 58:2 (1999), 326-335.

[49] P. Pudlák, "Lower bounds for resolution and cutting plane proofs and monotone computations", J. Symbolic Logic, 62:3 (1997), 981-998.

[50] J. Kóllar, L. Rónyai, T. Szabó, "Norm-graphs and bipartite Turán numbers", Combinatorica, 16:3 (1996), 399-406.

[51] D. J. Kleitman, "Extremal properties of collections of subsets containing no two sets and their union", J. Combinatorial Theory Ser. A, 20:3 (1976), 390-392.

[52] K. O'Bryant, "A complete annotated bibliography of work related to Sidon sequences", Electron. J. Combin., 11 (2004).

[53] V. Nikiforov, "A contribution to the Zarankiewicz problem", Linear Algebra Appl., 432:6 (2010), 1405-1411.

[54] Z. Füredi, "An upper bound on Zarankiewicz' problem", Combin. Probab. Comput., 5:1 (1996), 29-33.

[55] М. Холл, Комбинаторика, Мир, М., 1970; пер. с англ.: М. Hall, Combinatorial theory, Blaisdell Publ., Waltham, MA-Toronto-London, 1967.

[56] В.Е. Алексеев, “Две конструкции разностных множеств”, Проблемы кибернетики, т. 38, Наука, М., 1981, 259-262.

[57] J.B. Rosser, L. Schoenfeld, "Approximate formulas for some functions of prime numbers", Illinois J. Math., 6 (1962), 64-94.

[58] У. Г. Браун, "Графы, не содержащие графа Томсена", Кибернетический сборник. Новая серия, т. 18, Мир, М., 1981, 34-38; пер. с англ.: W. G. Brown, "On graphs that do not contain a Thomsen graph", Canad. Math. Bull., 9 (1966), 281-285.

[59] З. И. Боревич, И. Р. Шафаревич, Теория чисел, 3-е изд., Наука, М., 1985; англ. пер. 1-го изд.: А. I. Borevich, I. R. Shafarevich, Number theory, Academic Press, New York-London, 1966.

[60] С. Б. Гашков, И. С. Сергеев, "О применении метода аддитивных цепочек к инвертированию в конечных полях", Дискрет. матем., 18:4 (2006), 56-72; англ. 
пер.: S. B. Gashkov, I.S. Sergeev, "An application of the method of additive chains to inversion in finite fields", Discrete Math. Appl., 16:6 (2006), 601-618.

[61] N. Pippenger, "On the evaluation of powers and monomials", SIAM J. Comput., 9:2 (1980), 230-250.

[62] П. Эрдеш, Дж. Спенсер, Вероятностные методы в комбинаторике, Мир, М., 1976; пер. с англ.: P. Erdős, J. Spencer, Probabilistic methods in combinatorics, Wiley-Intersci. Ser. Discrete Math. Optim., Academic Press, New York-London, 1974.

[63] А.Е. Андреев, "Об одном семействе булевых матриц", Вестн. Моск. ун-та. Сер. 1. Матем., мех., 1986, № 2, 97-100; англ. пер.: А. E. Andreev, "On a family of Boolean matrices", Moscow Univ. Math. Bull., 41:2 (1986), 79-82.

[64] С. Б. Гашков, И. С. Сергеев, "О сложности линейных булевых операторов с редкими матрицами", Дискретн. анализ и исслед. опер., 17:3 (2010), 3-18; англ. пер.: S. B. Gashkov, I.S. Sergeev, "On the complexity of linear Boolean operators with thin matrices", J. Appl. Ind. Math., 5:2 (2011), 202-211.

[65] М. И. Гринчук, "О сложности реализации циклических булевых матриц вентильными схемами”, Изв. вузов. Матем., 1988, № 7, 39-44; англ. пер.: M. I. Grinchuk, "Complexity of implementing cyclic Boolean matrices by means of gate circuits", Soviet Math. (Iz. VUZ), 32:7 (1988), 65-72.

[66] М. И. Гринчук, И. С. Сергеев, "Редкие циркулянтные матрицы и нижние оценки сложности некоторых булевых операторов”, Дискретн. анализ и исслед. опер., 18:5 (2011), 38-53.

[67] К. Мельхорн, "Некоторые замечания, касающиеся булевых сумм”, Кибернетический сборник. Новая серия, т. 18, Мир, М., 1981, 39-45; пер. с англ.: K. Mehlhorn, "Some remarks on Boolean sums", Acta Inf., 12:4 (1979), 371-375.

[68] С. Б. Гашков, И. Б. Гашков, "О сложности вычисления дифференциалов и градиентов", Дискрет. матем., 17:3 (2005), 45-67; англ. пер.: S. B. Gashkov, I. B. Gashkov, "On the complexity of calculation of differentials and gradients", Discrete Math. Appl., 15:4 (2005), 327-350.

[69] А. Ахо, Дж. Хопкрофт, Дж. Ульман, Проектирование и анализ вычислительных алгоритмов, Мир, М., 1979; пер. с англ.: А. V. Aho, J. E. Hopcroft, J. D. Ullman, The design and analysis of computer algorithms, Addison-Wesley, Reading, MA, 1974.

[70] A. Shpilka, A. Yehudayoff, "Arithmetic circuits: a survey of recent results and open questions", Found. Trends Theor. Comput. Sci., 5:3-4 (2010), 207-388.

[71] I. Wegener, The complexity of Boolean functions, Wiley-Teubner Ser. Comput. Sci., Wiley, Stuttgart, 1987.

[72] N. Blum, "On negations in Boolean networks", Efficient algorithms, Lecture Notes in Comput. Sci., 5760, Springer-Verlag, Berlin-Heidelberg, 2009, 18-29.

\section{C. Б. Гашков (S. B. Gashkov)}

Механико-математический факультет

Московского государственного университета

им. М. В. Ломоносова

E-mail: sbgashkov@gmail.com

\section{И. С. Сергеев (I. S. Sergeev)}

Механико-математический факультет

Московского государственного университета

им. М. В. Ломоносова

E-mail: isserg@gmail.com
Поступила в редакцию 29.06.2011 и 11.04 .2012 